\title{
Cosmology in ghost-free bigravity theory with twin matter fluids: The origin of "dark matter"
}

\author{
Katsuki AOKI ${ }^{1, *}$ AND Kei-ichi MAEDA ${ }^{1, \text {, }}$ \\ 1 Department of Physics, Waseda University, Shinjuku, Tokyo 169-8555, Japan
}

(Dated: September 1, 2021)

\begin{abstract}
We study dynamics of Friedmann-Lemaitre-Robertson-Walker (FLRW) spacetime based on the ghost-free bigravity theory. Assuming the coupling parameters guaranteeing the existence of de Sitter space as well as Minkowski spacetime, we find two stable attractors for spacetime with "twin" dust matter fields: One is de Sitter accelerating universe and the other is matter dominated universe. Although a considerable number of initial data leads to de Sitter universe, we also find matter dominated universe or spacetime with a future singularity for some initial data. The cosmic nohair conjecture does not exactly hold, but the accelerating expansion can be found naturally. The $\Lambda$-CDM model is obtained as an attractor. We also show that the dark matter component in the Friedmann equation, which originates from another twin matter, can be about 5 times larger than the baryonic matter, by choosing the appropriate coupling constants.
\end{abstract}

PACS numbers:

\section{INTRODUCTION}

Recent observation has confirmed the big bang scenario of the expanding universe. The cosmological parameters are determined very precisely [1]. Cosmology is now precision science. However those observations reveal new unsolved problems in cosmology; dark energy and dark matter. Dark matter could be explained by unknown elementary particles, although there may be other possibilities. On the other hand, dark energy, which is the origin of the current accelerated expansion of the Universe, is one of the biggest mysteries in modern cosmology [2, 3$]$. The acceleration might be due to some unknown matter with a strange equation of state, or might be due to a modification of general relativity (GR). In this paper, we are interested in the latter possibility. Among many modified gravity theories, one natural modification of GR is to consider the possibility of a massive graviton. The first attempt to consider a massive graviton was proposed by Fierz and Pauli [4]. Although a simple non-linear extension of the Fierz-Pauli massive gravity theory contains instabilities called the Boulware-Deser ghost [5], it was recently shown that the special choice of the interaction term can exclude such a ghost state [6 11] 12]. However, this theory cannot describe the flat Friedmann universe, if the fictitious metric for the Stückelberg field is Minkowski's one. One may consider an inhomogeneous metric or extend it to de Sitter metric. If we discuss an curved fictitious geometry, it may be natural for it to be dynamical. In fact the dRGT massive gravity theory has been generalized to such a bigravity theory, which is still ghost-free. It contains a massless spin- 2 particle and a

\footnotetext{
*Electronic address: katsuki-a12@gravity.phys.waseda.ac.jp

${ }^{\dagger}$ Electronic address: maeda@waseda.ac.jp
}

massive spin-2 particle [13].

Such theories with a massive graviton are also motivated by the dark energy problem. The accelerating universe may be phenomenologically described by the $\Lambda$ CDM model. However, the theoretically expected value of a cosmological constant (the vacuum expectation value of some fields) is too large to explain the observed value of dark energy. In the massive gravity theory, the nonlinear "mass term" gives an effective cosmological constant. So the graviton mass is the scale of the Hubble expansion rate, dark energy could be explained by the massive gravity theory.

Unfortunately, in the massive gravity, a flat Friedmann universe with a fiducial Minkowski metric cannot be a solution. Only an open Friedmann universe solves the basic equations [14, 23]. In order to find a flat Friedmann universe, the fiducial metric should be more generic. One possibility is to assume an isotropic and inhomogeneous metric form [16 20]. However, the coupling constants are restricted in this model to find an accelerating universe. The other possibility is that the fiducial spacetime is no longer the Minkowski's one, but it is assumed to be a curved spacetime. If one choose de Sitter spacetime, we find a flat (or closed) Friedmann universe as well as an open universe. However, if both metrics are homogeneous and isotropic, there appears a new type of ghost instability. An anisotropic fiducial metric may provide a stable flat Friedmann universe 2123 .

If the fiducial spacetime is a curved spacetime, it may be natural for its metric to be dynamical as well. Hence, based on a ghost-free bigravity theory, cosmological models are also studied [24 34]. In contrast to the massive gravity theory, these exists a self-accelerating solution for all type Freedmann universe in the bigravity theory.

The bigravity theory includes GR with/without a cosmological constant as a special case. If both metric are proportional, which we call a homothetic solution, the 
basic equations are reduced to two sets of the Einstein equations with cosmological constants, which originate from the interaction terms of two metrics 35]. When the cosmological constant is positive, we have a chance to find de Sitter accelerating universe since the vacuum solution is de Sitter spacetime.

Although a considerable literature on bigravity has discussed on the possibility of the accelerating universe, it has mainly dealt with the case such that the matter field interacts only with our physical metric. A little attention has been paid to the effect from an "exotic" matter which interacts with another metric. Note that if matter fields interact with both metrics, it will violate the equivalence principle, which holds in very high accuracy [36]. Hence we have to discuss two different matter fields, which are decoupled each other and interact only through two metric interactions. We then call them twin matter fields 37 ].

Since GR is consistent with many experiments and observations [36], homothetic solutions given by those in GR may be preferred in the bigravity theory as well. However, in homothetic solutions two matters must satisfy a fine tuned condition [27]. Hence we have to include another twin matter as well as our matter field in the dynamics of bigravity and discuss whether we obtain a homothetic solution as an attractor.

The purpose of this study is to investigate cosmology in the ghost-free bigravity furthermore. We consider both metric are described by the FLRW metrics and each metric interacts with one of twin matter fields, respectively. We assume both are ordinary matters, which energymomenta are conserved individually. However we do not assume that twin matter fields satisfy a fine tuned condition for homothetic solutions.

Since a vacuum homothetic solution can be de Sitter, we will analyze whether this de Sitter accelerating universe is found as an attractor. The result is related to the so called cosmic ho hair conjecture [38 44], in which all expanding universes with a positive cosmological constant is found asymptotically approach the de Sitter spacetime. If it is the case, it will guarantee naturalness of accelerating expansion in the bigravity theory. The other interesting point in the present analysis is whether another twin matter field behaves as dark matter. If we find de Sitter universe with twin dark matter as an attractor in the present model, the $\Lambda$-CDM cosmological model, which is phenomenologically favored from observations [1], is obtained naturally from the bigravity theory.

The paper is organized as follows. Introducing the ghost-free bigravity, we summarize the basic equations and present a homothetic solution in section II. In section III. we show the Friedmann equations in bigravity theory for the coupling constants which guarantee the existence of the Minkowski spacetime, and present the vacuum solutions. We the analyze the dynamics of the Friedmann equations in section IV We show that the fate of the uni- verse is classified into three types (the self-accelerating de Sitter spacetime, the decelerating matter dominant universe, and the universe with a future singularity). The de Sitter spacetime is found from natural initial conditions. In section $\mathrm{V}$, we study whether we can find an observationally consistent model in our cosmological solutions. We find that the $\Lambda$-CDM model is obtained as an attractor, and dark matter can be explained by another twin matter for the appropriate coupling constants. We summarize our results and give some remarks in section VI.

\section{BIGRAVITY THEORY}

\section{A. Hassan-Rosen bigravity model}

In the present papers, we will focus only on the ghostfree bigravity theory, although many bigravity theories have been proposed [45 50]. The ghost-free bigravity theory proposed by Hassan and Rosen [13] is described by the action

$$
\begin{aligned}
S & =\frac{1}{2 \kappa_{g}^{2}} \int d^{4} x \sqrt{-g} R(g)+\frac{1}{2 \kappa_{f}^{2}} \int d^{4} x \sqrt{-f} \mathcal{R}(f) \\
& +S^{[\mathrm{m}]}\left(g, f, \psi_{g}, \psi_{f}\right)-\frac{m^{2}}{\kappa^{2}} \int d^{4} x \sqrt{-g} \mathscr{U}(g, f)(2
\end{aligned}
$$

where $g_{\mu \nu}$ and $f_{\mu \nu}$ are two dynamical metrics, and $R(g)$ and $\mathcal{R}(f)$ are those Ricci scalars, respectively. $\kappa_{g}^{2}=8 \pi G$ and $\kappa_{f}^{2}=8 \pi \mathcal{G}$ are the corresponding gravitational couplings, while $\kappa$ is defined by $\kappa^{2}=\kappa_{g}^{2}+\kappa_{f}^{2}$. We assume that the matter action $S^{(\mathrm{m})}$ is divided into two parts:

$$
S^{(\mathrm{m})}\left(g, f, \psi_{g}, \psi_{f}\right)=S_{g}^{[\mathrm{m}]}\left(g, \psi_{g}\right)+S_{f}^{[\mathrm{m}]}\left(f, \psi_{f}\right),
$$

i.e., matter fields $\psi_{g}$ and $\psi_{f}$ are coupled only to $g$-metric and to $f$-metric, respectively. This restriction guarantees the equivalence principle. We call $\psi_{g}$ (g-matter) and $\psi_{f}$ ( $f$-matter) twin matter fluids.

The interaction term between two metrics is given by

$$
\mathscr{U}(g, f)=\sum_{k=0}^{4} b_{k} \mathscr{U}_{k}(\gamma)=1,
$$

$$
\begin{aligned}
& \mathscr{U}_{0}(\gamma)=-\frac{1}{4 !} \epsilon_{\mu \nu \rho \sigma} \epsilon^{\mu \nu \rho \sigma}, \\
& \mathscr{U}_{1}(\gamma)=-\frac{1}{3 !} \epsilon_{\mu \nu \rho \sigma} \epsilon^{\alpha \nu \rho \sigma} \gamma_{\alpha}^{\mu}, \\
& \mathscr{U}_{2}(\gamma)=-\frac{1}{4} \epsilon_{\mu \nu \rho \sigma} \epsilon^{\alpha \beta \rho \sigma} \gamma^{\mu}{ }_{\alpha} \gamma_{\beta}^{\nu}, \\
& \mathscr{U}_{3}(\gamma)=-\frac{1}{3 !} \epsilon_{\mu \nu \rho \sigma} \epsilon^{\alpha \beta \gamma \sigma} \gamma_{\alpha}^{\mu} \gamma^{\nu}{ }_{\beta} \gamma_{\gamma}, \\
& \mathscr{U}_{4}(\gamma)=-\frac{1}{4 !} \epsilon_{\mu \nu \rho \sigma} \epsilon^{\alpha \beta \gamma \delta} \gamma_{\alpha}^{\mu} \gamma_{\beta}{ }_{\beta} \gamma_{\gamma}{ }_{\gamma} \gamma_{\delta}^{\sigma},
\end{aligned}
$$


where $b_{k}$ are coupling constants, while $\gamma_{\nu}^{\mu}$ is defined by

$$
\gamma_{\rho}^{\mu} \gamma_{\nu}^{\rho}=g^{\mu \rho} f_{\rho \nu}
$$

In order to take the square root to obtain the explicit form of $\gamma^{\mu}{ }_{\nu}$, we shall introduce the tetrad systems, $\left\{e_{\mu}^{(a)}\right\}$ and $\left\{\omega_{\mu}^{(a)}\right\}$, which are defined by

$$
g_{\mu \nu}=\eta_{a b} e_{\mu}^{(a)} e_{\nu}^{(b)}, \quad f_{\mu \nu}=\eta_{a b} \omega_{\mu}^{(a)} \omega_{\nu}^{(b)},
$$

with an additional constraint $e^{\mu}{ }_{(a)} \omega_{\mu(b)}=e^{\mu}{ }_{(b)} \omega_{\mu(a)}$. This constraint guarantees that the tetrad description is equivalent to the metric description.

We then find

$$
\gamma_{\nu}^{\mu}=\epsilon \eta_{a b} e^{\mu(a)} \omega_{\nu}^{(b)}
$$

where $\epsilon= \pm 1$ comes from the square root. As for the directions of tetrads, we choose that $e_{\mu}^{(0)} d x^{\mu}$ and $\omega_{\mu}^{(0)} d x^{\mu}$ are future-directed for $d t>0$. Changing the sign of $\epsilon$ corresponds to the following transformation

$$
\gamma_{\nu}^{\mu} \leftrightarrow-\gamma_{\nu}^{\mu}
$$

for which the interaction term is invariant by changing the sign of the coupling constants as

$$
b_{k} \leftrightarrow(-1)^{k} b_{k} \quad(k=0-4) .
$$

Note that $\mathscr{U}_{0}(\gamma)$ and $\mathscr{U}_{4}(\gamma)$ do not contribute to the equations of motion for $f_{\mu \nu}$ and $g_{\mu \nu}$, respectively, because

$$
\begin{aligned}
& \sqrt{-g} \mathscr{U}_{0}(\gamma)=\sqrt{-g} \\
& \sqrt{-g} \mathscr{U}_{4}(\gamma)=\sqrt{-f} .
\end{aligned}
$$

which are just cosmological constants in $g$-spacetime and $f$-spacetime [51], respectively. The interaction term is also written by another tensor defined by $\mathcal{K}_{\nu}^{\mu}=\delta_{\nu}^{\mu}-\gamma^{\mu}{ }_{\nu}$ as

$$
\mathscr{U}(g, f)=\sum_{k=0}^{4} c_{k} \mathscr{U}_{k}(\mathcal{K})
$$

where the relations between $\left\{b_{k}\right\}$ and $\left\{c_{k}\right\}$ are

$$
\begin{aligned}
& c_{0}=b_{0}+4 b_{1}+6 b_{2}+4 b_{3}+b_{4}, \\
& c_{1}=-\left(b_{1}+3 b_{2}+3 b_{3}+b_{4}\right), \\
& c_{2}=b_{2}+2 b_{3}+b_{4}, \\
& c_{3}=-\left(b_{3}+b_{4}\right), \\
& c_{4}=b_{4} .
\end{aligned}
$$

If we require a flat Minkowski spacetime to be a solution of the field equations, we have to impose the following condition

$$
c_{0}=c_{1}=0
$$

Since $m$ is assumed to be the mass of graviton in the Minkowski background in massive gravity limit, we should set

$$
c_{2}=-1
$$

This quadratic term $\mathscr{U}_{2}(\mathcal{K})$ gives the Fierz-Pauli term in the limit of linear massive gravity theory [4].

As a result, $\left\{b_{k}\right\}$ are also given by two free coupling constants $c_{3}$ and $c_{4}$ as

$$
\begin{aligned}
& b_{0}=4 c_{3}+c_{4}-6, \\
& b_{1}=3-3 c_{3}-c_{4}, \\
& b_{2}=2 c_{3}+c_{4}-1, \\
& b_{3}=-\left(c_{3}+c_{4}\right), \\
& b_{4}=c_{4} .
\end{aligned}
$$

In the present paper, we shall focus on this choice of the coupling constants except for some special case such as the partially massless theory.

In de Sitter spacetime, it is known that there exists the so-called Higuchi bound

$$
m_{F P}^{2}=\frac{2}{3} \Lambda,
$$

where $m_{F P}$ is the mass of spin- 2 particle in the linear massive gravity theory [4] and $\Lambda$ is a cosmological constant. Beyond this bound, no ghost appears and then five modes of the massive graviton can propagate properly, while below the bound, the helicity-zero mode becomes a ghost [52, 53]. At the exact bound value, however, the helicity-zero mode is decoupled and a new gauge symmetry appears. Such a theory is often referred to partially massless (PM). A non-linear extension is known as the PM massive gravity, and the extension to bigravity theory (the PM bimetric theory) is also discussed [54, 55]. These PM theories are characterized by the coupling constants such that

$$
b_{1}=b_{3}=0, \quad b_{0}=3 b_{2} \kappa_{f}^{2} / \kappa_{g}^{2}, \quad b_{4}=3 b_{2} \kappa_{g}^{2} / \kappa_{f}^{2} .
$$

We shall discuss this special case separately in Appendix.

\section{B. The equations of motion}

Taking the variation of the action with respect to $g_{\mu \nu}$ and $f_{\mu \nu}$, we find two sets of the Einstein equations:

$$
\begin{aligned}
G^{\mu}{ }_{\nu} & =\kappa_{g}^{2}\left(T^{[\gamma] \mu}{ }_{\nu}+T^{[\mathrm{m}] \mu}{ }_{\nu}\right), \\
\mathcal{G}^{\mu}{ }_{\nu} & =\kappa_{f}^{2}\left(\mathcal{T}^{[\gamma] \mu}{ }_{\nu}+\mathcal{T}^{[\mathrm{m}] \mu}{ }_{\nu}\right),
\end{aligned}
$$

where $G^{\mu}{ }_{\nu}$ and $\mathcal{G}^{\mu}{ }_{\nu}$ are the Einstein tensors for $g_{\mu \nu}$ and $f_{\mu \nu}$, respectively. The matter energy-momentum tensors 
are given by

$$
\begin{aligned}
T_{\mu \nu}^{[\mathrm{m}]} & =-2 \frac{\delta S_{g}^{[\mathrm{m}]}}{\delta g^{\mu \nu}} \\
\mathcal{T}_{\mu \nu}^{[\mathrm{m}]} & =-2 \frac{\delta S_{f}^{[\mathrm{m}]}}{\delta f^{\mu \nu}} .
\end{aligned}
$$

The energy-momentum tensors of "gravitons", which are from the interaction term, are given by

$$
\begin{aligned}
T_{\nu}^{[\gamma] \mu} & =\frac{m^{2}}{\kappa^{2}}\left(\tau^{\mu}{ }_{\nu}-\mathscr{U} \delta^{\mu}{ }_{\nu}\right), \\
\mathcal{T}^{[\gamma] \mu}{ }_{\nu} & =-\frac{\sqrt{-g}}{\sqrt{-f}} \frac{m^{2}}{\kappa^{2}} \tau^{\mu}{ }_{\nu},
\end{aligned}
$$

with

$$
\begin{aligned}
\tau_{\nu}^{\mu} & =\left\{b_{1} \mathscr{U}_{0}+b_{2} \mathscr{U}_{1}+b_{3} \mathscr{U}_{2}+b_{4} \mathscr{U}_{3}\right\} \gamma^{\mu}{ }_{\nu} \\
& -\left\{b_{2} \mathscr{U}_{0}+b_{3} \mathscr{U}_{1}+b_{4} \mathscr{U}_{2}\right\}\left(\gamma^{2}\right)^{\mu}{ }_{\nu} \\
& +\left\{b_{3} \mathscr{U}_{0}+b_{4} \mathscr{U}_{1}\right\}\left(\gamma^{3}\right)^{\mu}{ }_{\nu} \\
& -b_{4} \mathscr{U}_{0}\left(\gamma^{4}\right)^{\mu}{ }_{\nu} .
\end{aligned}
$$

The energy-momenta of matter fields are assumed to be conserved individually as

$$
\stackrel{(g)}{\nabla}_{\mu} T^{[\mathrm{m}] \mu}{ }_{\nu}=0, \stackrel{(f)}{\nabla}_{\mu} \mathcal{T}^{[\mathrm{m}] \mu}{ }_{\nu}=0
$$

where $\stackrel{(g)}{\nabla}_{\mu}$ and $\stackrel{(f)}{\nabla}_{\mu}$ are covariant derivatives with respect to $g_{\mu \nu}$ and $f_{\mu \nu}$. From the contracted Bianchi identities for 2.20) and (2.21), the conservation of energymomenta for "gravitons" are also guaranteed as

$$
\stackrel{(g)}{\nabla}_{\mu} T^{[\gamma] \mu}{ }_{\nu}=0, \stackrel{(f)}{\nabla}_{\mu} \mathcal{T}^{[\gamma] \mu}{ }_{\nu}=0
$$

\section{Homothetic solution}

Before going to discuss cosmology, first we give one simple solution, in which we assume that two metrics are proportional;

$$
f_{\mu \nu}=K^{2} g_{\mu \nu},
$$

where $K$ is a scalar function. In this case, since we find the tensor $\gamma^{\mu}{ }_{\nu}=K \delta^{\mu}{ }_{\nu}$, the energy-momentum tensors from the interaction term is given by

$$
\begin{aligned}
\kappa_{g}^{2} T^{[\gamma] \mu}{ }_{\nu} & =-\Lambda_{g}(K) \delta^{\mu}{ }_{\nu}, \\
\kappa_{f}^{2} \mathcal{T}^{[\gamma] \mu}{ }_{\nu} & =-\Lambda_{f}(K) \delta^{\mu}{ }_{\nu},
\end{aligned}
$$

where

$$
\begin{aligned}
& \Lambda_{g}(K)=m_{g}^{2}\left(b_{0}+3 b_{1} K+3 b_{2} K^{2}+b_{3} K^{3}\right) \\
& \Lambda_{f}(K)=m_{f}^{2}\left(b_{1}+3 b_{2} K^{-1}+3 b_{3} K^{-2}+b_{4} K^{-3}\right)
\end{aligned}
$$

with

$$
\begin{gathered}
m_{g}^{2}=\frac{\kappa_{g}^{2}}{\kappa^{2}} m^{2}, \text { and } m_{f}^{2}=\frac{\kappa_{f}^{2}}{\kappa^{2}} m^{2} \\
\left(m_{g}^{2}+m_{f}^{2}=m^{2}\right) .
\end{gathered}
$$

From the energy-momentum conservation (2.26), we find that $K$ is a constant. As a result, we find two sets of the Einstein equations with cosmological constants $\Lambda_{g}$ and $\Lambda_{f}$ :

$$
\begin{aligned}
G_{\mu \nu}(g)+\Lambda_{g} g_{\mu \nu} & =\kappa_{g}^{2} T^{[\mathrm{m}]}{ }_{\mu \nu} \\
\mathcal{G}_{\mu \nu}(f)+\Lambda_{f} f_{\mu \nu} & =\kappa_{f}^{2} \mathcal{T}^{[\mathrm{m}]}{ }_{\mu \nu} .
\end{aligned}
$$

Since two metrics are proportional, we have the constraints on the cosmological constants and matter fields as

$$
\begin{aligned}
& \Lambda_{g}(K)=K^{2} \Lambda_{f}(K) \\
& \mathcal{T}_{\mu \nu}^{[\mathrm{m}]}=K^{2} T_{\mu \nu}^{[\mathrm{m}]} .
\end{aligned}
$$

Since (2.31) is a quartic equation of $K$, we have at most four real roots of $K$, which give four different cosmological constants. The basic equations (2.29) (or (2.30) are just the Einstein equations in GR with a cosmological constant. Hence any solutions in GR with a cosmological constant are always the solutions in the present bigravity theory. We shall call these solutions homothetic solutions because of the proportionality of two metrics.

If we assume a flat Minkowski spacetime is one of the solutions in the case of vacuum state, that is, if the coupling constants are given by (2.14) and (2.15), or (2.17), we find $K=1$ is always one of the solutions, which gives zero cosmological constant $\left(\Lambda_{g}(1)=\Lambda_{f}(1)=0\right)$. Among the rest three solutions of $K$, if we find a positive cosmological constant, we obtain an accelerating expansion of the universe, which evolves into de Sitter solution, if the cosmic ho hair conjecture holds.

In Appendix, we present the perturbation equations for homothetic solutions. The mass of massive mode in the homothetic background is given by

$$
m_{\mathrm{eff}}^{2}=\left(m_{g}^{2}+\frac{m_{f}^{2}}{K^{2}}\right)\left(b_{1} K+2 b_{2} K^{2}+b_{3} K^{3}\right) .
$$

The homothetic de Sitter solution is stable against linear perturbations.

\section{COSMOLOGY IN BIGRAVITY}

Based on the ghost-free bigravity theory as well as the massive gravity theory, many authors have studied cosmological models. In this paper, we analyze the details of the evolution of the universe including both matter fields and study whether an accelerating expansion is naturally 
found in the late time. This is related to the so-called cosmic no hair conjecture in general relativity (GR), in which de Sitter solution is an attractor for generic initial conditions if there exists a cosmological constant. Especially, we focus on the effect of matter fields including $f$-matter, which has not been studied so much.

\section{A. FLRW universe}

Now we discuss the FLRW spacetime, which metrics are given by $[56$ ]

$$
\begin{aligned}
& d s_{g}^{2}=-N_{g}^{2}(t) d t^{2}+a_{g}^{2}(t)\left(\frac{d r^{2}}{1-k r^{2}}+r^{2} d \Omega^{2}\right) \\
& d s_{f}^{2}=-N_{f}^{2}(t) d t^{2}+a_{f}^{2}(t)\left(\frac{d r^{2}}{1-k r^{2}}+r^{2} d \Omega^{2}\right)
\end{aligned}
$$

where $N_{g}$ and $N_{f}$ are lapse functions, while $a_{g}$ and $a_{f}$ are scale factors for $g_{\mu \nu}$ and $f_{\mu \nu}$, respectively. Since those variables must be positive, we choose the tetrads as

$$
\begin{gathered}
\left\{e_{\mu}^{(a)}\right\}=\operatorname{diag}\left(N_{g}, \frac{a_{g}}{\sqrt{1-k r^{2}}}, a_{g}, a_{g} \sin \theta\right) \\
\left\{\omega_{\mu}^{(a)}\right\}=\operatorname{diag}\left(N_{f}, \frac{a_{f}}{\sqrt{1-k r^{2}}}, a_{f}, a_{f} \sin \theta\right)
\end{gathered}
$$

Hence the interaction tensor is given by

$$
\gamma_{\nu}^{\mu}=\epsilon \operatorname{diag}(A, B, B, B),
$$

where $A=N_{f} / N_{g}$ and $B=a_{f} / a_{g}$.

The cosmic times for $g$ - and $f$-metrics are defined by

$$
\tau_{g}=\int N_{g}(t) d t, \quad \tau_{f}=\int N_{f}(t) d t
$$

respectively. Using the gauge freedom, in what follows, we set $N_{g}=1$, in which gauge choice, the time coordinate $t$ is the same as the cosmic time of $g$-metric.

Setting $\tilde{A}=\epsilon A, \tilde{B}=\epsilon B$, we find that the interaction energy-momentum tensors are given by

$$
\begin{aligned}
& T_{g}^{[\gamma] \mu}=\operatorname{diag}\left[-\rho_{g}^{[\gamma]}, P_{g}^{[\gamma]}, P_{g}^{[\gamma]}, P_{g}^{[\gamma]]},\right. \\
& T_{f}^{[\gamma] \mu}=\operatorname{diag}\left[-\rho_{f}^{[\gamma]}, P_{f}^{[\gamma]}, P_{f}^{[\gamma]}, P_{f}^{[\gamma]}\right]
\end{aligned}
$$

where

$$
\begin{aligned}
\rho_{g}^{[\gamma]}= & \frac{m^{2}}{\kappa^{2}}\left(b_{0}+3 b_{1} \tilde{B}+3 b_{2} \tilde{B}^{2}+b_{3} \tilde{B}^{3}\right), \\
P_{g}^{[\gamma]}=- & \frac{m^{2}}{\kappa^{2}}\left[b_{0}+b_{1}(\tilde{A}+2 \tilde{B})\right. \\
& \left.\quad+b_{2}\left(2 \tilde{A} \tilde{B}+\tilde{B}^{2}\right)+b_{3} \tilde{A} \tilde{B}^{2}\right], \\
\rho_{f}^{[\gamma]}= & \frac{m^{2}}{\kappa^{2}}\left(b_{4}+\frac{3 b_{3}}{\tilde{B}}+\frac{3 b_{2}}{\tilde{B}^{2}}+\frac{b_{1}}{\tilde{B}^{3}}\right), \\
P_{f}^{[\gamma]}= & -\frac{m^{2}}{\kappa^{2}}\left[b_{4}+b_{3}\left(\frac{1}{\tilde{A}}+\frac{2}{\tilde{B}}\right)\right. \\
& \left.\quad+b_{2}\left(\frac{2}{\tilde{A} \tilde{B}}+\frac{1}{\tilde{B}^{2}}\right)+\frac{b_{1}}{\tilde{A} \tilde{B}^{2}}\right] .
\end{aligned}
$$

We assume that twin matter fields ( $g$-matter and $f$ matter fluids) are described by perfect fluids:

$$
\begin{aligned}
& T_{g}^{[\mathrm{m}] \mu}{ }_{\nu}=\operatorname{diag}\left[-\rho_{g}(t), P_{g}(t), P_{g}(t), P_{g}(t)\right], \\
& T_{f}^{[\mathrm{m}] \mu}=\operatorname{diag}\left[-\rho_{f}(t), P_{f}(t), P_{f}(t), P_{f}(t)\right] .
\end{aligned}
$$

Assume that the universe consists of dust (nonrelativistic matter) and radiation (relativistic matter) for twin matter fluids. From the conservation equations,

$$
\begin{aligned}
& \dot{\rho}_{g}+3 \frac{\dot{a}_{g}}{a_{g}}\left(\rho_{g}+P_{g}\right)=0, \\
& \dot{\rho}_{f}+3 \frac{\dot{a}_{f}}{a_{f}}\left(\rho_{f}+P_{f}\right)=0,
\end{aligned}
$$

where the dot denotes the derivative with respect to $t$, the energy densities are described by the scale factors as

$$
\begin{aligned}
& \kappa_{g}^{2} \rho_{g}=\kappa_{g}^{2}\left(\rho_{g, \mathrm{~m}}+\rho_{g, \mathrm{r}}\right)=\frac{c_{g, \mathrm{~m}}}{a_{g}^{3}}+\frac{c_{g, \mathrm{r}}}{a_{g}^{4}} \\
& \kappa_{f}^{2} \rho_{f}=\kappa_{f}^{2}\left(\rho_{f, \mathrm{~m}}+\rho_{f, \mathrm{r}}\right)=\frac{c_{f, \mathrm{~m}}}{a_{f}^{3}}+\frac{c_{f, \mathrm{r}}}{a_{f}^{4}},
\end{aligned}
$$

where $c_{g, \mathrm{~m}}, c_{g, \mathrm{r}}, c_{f, \mathrm{~m}}$ and $c_{f, \mathrm{r}}$ are positive integration constants.

The Einstein equations with the metric ansatz (3.1) and (3.2) are reduced to the Friedmann equations:

$$
\begin{aligned}
& H_{g}^{2}+\frac{k}{a_{g}^{2}}=\frac{\kappa_{g}^{2}}{3}\left[\rho_{g}^{[\gamma]}+\rho_{g}\right], \\
& H_{f}^{2}+\frac{k}{a_{f}^{2}}=\frac{\kappa_{f}^{2}}{3}\left[\rho_{f}^{[\gamma]}+\rho_{f}\right],
\end{aligned}
$$

where

$$
H_{g}=\frac{\dot{a}_{g}}{a_{g}}, \quad H_{f}=\frac{\dot{a}_{f}}{N_{f} a_{f}}
$$

are the Hubble expansion parameters.

The conservation equations for $T_{g}^{[\gamma] \mu}{ }_{\nu}$ and $T_{f}^{[\gamma] \mu}{ }_{\nu}$ are reduced to one equation:

$$
\left(\frac{\dot{a_{f}}}{\dot{a_{g}}}-A\right)\left(b_{1}+2 b_{2} \tilde{B}+b_{3} \tilde{B}^{2}\right)=0 .
$$

These are two cases: The first parentheses vanishes or the second one does so. If the second parentheses vanishes, $\tilde{B}$ is a constant, and then $\rho_{g}^{[\gamma]}(\tilde{B})$ and $\rho_{f}^{[\gamma]}(\tilde{B})$ are also constant. As a result, the Friedmann equations 3.15) and $(3.16$ ) are the same as the ordinary ones in GR with a cosmological constant. Since the evolution of the universe is well analyzed in GR, we will not discuss this case furthermore.

Thus, we assume that the first parentheses vanishes. This condition holds when

$$
H_{g}=B H_{f}
$$


From two Friedmann equations (3.15), 3.16) with the condition (3.19), we find one algebraic equation

$$
\kappa_{g}^{2}\left[\rho_{g}^{[\gamma]}(\tilde{B})+\rho_{g}\left(a_{g}\right)\right]-\kappa_{f}^{2} \tilde{B}^{2}\left[\rho_{f}^{[\gamma]}(\tilde{B})+\rho_{f}\left(a_{f}\right)\right]=0 .
$$

Since $a_{f}=B a_{g}=\epsilon \tilde{B} a_{g}$, this equation gives the relation between $\tilde{B}$ and $a_{g}$. It also provides us some information about the interaction term $\rho_{g}^{[\gamma]}$ and $\rho_{f}^{[\gamma]}$ in terms of twin matter fluids, which will be used in the discussion about dark matter later.

The above equation (3.20) with (3.14) is rewritten into a quartic equation for $a_{g}$ as

$$
\tilde{B} C_{\Lambda}(\tilde{B}) a_{g}^{4}+\tilde{B} C_{\mathrm{m}}(\tilde{B}) a_{g}+C_{\mathrm{r}}(\tilde{B})=0,
$$

where

$$
\begin{aligned}
C_{\Lambda}(\tilde{B})= & \tilde{B}\left[\kappa_{g}^{2} \rho_{g}^{[\gamma]}(\tilde{B})-\kappa_{f}^{2} \tilde{B}^{2} \rho_{f}^{[\gamma]}(\tilde{B})\right] \\
= & \kappa_{g}^{2} \tilde{B}\left(b_{3} \tilde{B}^{3}+3 b_{2} \tilde{B}^{2}+3 b_{1} \tilde{B}+b_{0}\right) \\
& -\kappa_{f}^{2}\left(b_{4} \tilde{B}^{3}+3 b_{3} \tilde{B}^{2}+3 b_{2} \tilde{B}+b_{1}\right) \\
C_{\mathrm{m}}(\tilde{B})= & c_{g, \mathrm{~m}} \tilde{B}-\epsilon c_{f, \mathrm{~m}} \\
C_{\mathrm{r}}(\tilde{B})= & c_{g, \mathrm{r}} \tilde{B}^{2}-c_{f, \mathrm{r}}
\end{aligned}
$$

Solving (3.21), we obtain the relation $a_{g}=a_{g}(\tilde{B})$ and then $a_{f}=\epsilon \tilde{B} a_{g}(\tilde{B})$. Plugging this relation into the Friedmann equation (3.15), we find the equation for $\tilde{B}$ as

$$
\left(\frac{d \tilde{B}}{d t}\right)^{2}+V_{g}(\tilde{B})=0
$$

where the potential for $\tilde{B}$ is given by

$V_{g}(\tilde{B})=\frac{a_{g}^{2}}{a_{g}^{\prime 2}}\left[\frac{k}{a_{g}^{2}(\tilde{B})}-\frac{1}{3}\left(\kappa_{g}^{2} \rho_{g}^{[\gamma]}(\tilde{B})+\frac{c_{g, \mathrm{~m}}}{a_{g}^{3}(\tilde{B})}+\frac{c_{g, \mathrm{r}}}{a_{g}^{4}(\tilde{B})}\right)\right]$

with

$$
a_{g}^{\prime}=-\frac{\left(C_{\Lambda}+\tilde{B} C_{\Lambda}^{\prime}\right) a_{g}^{4}+\left(2 c_{g, \mathrm{~m}} \tilde{B}-\epsilon c_{f, \mathrm{~m}}\right) a_{g}+2 c_{g, \mathrm{r}} \tilde{B}}{\tilde{B}\left(4 C_{\Lambda} a_{g}^{3}+C_{\mathrm{m}}\right)} .
$$

A prime denotes the derivative with respect to $\tilde{B}$.

\section{B. Vacuum solutions}

Since the matter energy densities drop as the universe expands, we may expect the spacetime evolves into a vacuum state asymptotically unless the spacetime encounters a singularity.

So before solving the dynamical equation (3.25), we first analyze the vacuum solutions. Eq. (3.21) is now simple as

$$
C_{\Lambda}(\tilde{B})=0
$$

This equation is the same as (2.31). Since $\tilde{B}$ is a constant, we find $A=B=K$, which gives the homothetic solution discussed in $\S$. IC.

We have at most four real roots $\tilde{B}_{\ell}(\ell=1, \cdots, 4)$ for Eq. (3.26). If we assume the existence of Minkowski space, we always have one trivial root $\tilde{B}_{(\mathrm{M})}=1\left(B_{(\mathrm{M})}=\right.$

\begin{tabular}{|c|c|c|c|c|c|c|}
\hline Model & $\left(c_{3}, c_{4}\right)$ & region & $\epsilon$ & $B_{\ell}$ & $\Lambda_{g}$ & vacuum \\
\hline \multirow[t]{4}{*}{ A } & \multirow[t]{4}{*}{$(-1,0)$} & \multirow[t]{4}{*}{ (1) } & -1 & 0.523476 & $-22.0323 m_{g}^{2}$ & $\mathrm{AdS}_{1}$ \\
\hline & & & 1 & 1 & 0 & $\mathrm{M}$ \\
\hline & & & 1 & 1.67319 & $-0.394464 m_{q}^{2}$ & $\overline{\mathrm{AdS}_{2}}$ \\
\hline & & & 1 & 6.85028 & $12.4267 \mathrm{~m}_{g}^{2}$ & $\mathrm{dS}$ \\
\hline \multirow[t]{4}{*}{ B } & \multirow[t]{4}{*}{$(4,-10)$} & \multirow[t]{4}{*}{$(2)$} & -1 & 1.91031 & $-80.4017 m_{g}^{2}$ & $\mathrm{AdS}_{1}$ \\
\hline & & & 1 & 1 & 0 & $\mathrm{M}$ \\
\hline & & & 1 & 0.145979 & $0.264813 m_{g}^{2}$ & $\mathrm{dS}$ \\
\hline & & & 1 & 0.59766 & $-0.140902 m_{g}^{2}$ & $\mathrm{AdS}_{2}$ \\
\hline
\end{tabular}
$1, \epsilon=1$ ), which gives zero cosmological constant. The rest three roots can be all real or one real and two complex.

First we shall look for de Sitter solution. When the following conditions are satisfied, we find one de Sitter solution, which is given by $B_{(\mathrm{dS})}$ with $\epsilon=1$ :

$$
\begin{array}{cc}
c_{3}<0, c_{3}+c_{4}<0,2 c_{3}^{2}+3 c_{4}>0 & {[\text { region }(1)],} \\
c_{3}>3,3 c_{3}+c_{4}<3,2 c_{3}^{2}+3 c_{4}>0 & {[\text { region }(2)] .}
\end{array}
$$

We show the typical examples (Models A and B) for these regions in Table I

TABLE I: In the parameter regions (1) and (2), there exists one de Sitter solution with $\tilde{B}_{(\mathrm{dS})}>0(\epsilon=1)$. In addition, we find three other vacuum solutions (two anti de Sitter (AdS) solutions as well as a trivial Minkowski spacetime). We assume $\kappa_{f}=\kappa_{g}$.

For the coupling constants which satisfy

$$
\begin{array}{ll}
c_{3}+c_{4}>0,3 c_{3}+c_{4}<3,2 c_{3}^{2}+3 c_{4}>0 & {[\operatorname{region}(3 \mathrm{a})]} \\
c_{3}+c_{4}<0,3 c_{3}+c_{4}>3,2 c_{3}^{2}+3 c_{4}>0 & {[\operatorname{region}(3 \mathrm{~b})]} \\
c_{3}+c_{4}>0,3 c_{3}+c_{4}>3,2 c_{3}^{2}+3 c_{4}>0 & {[\operatorname{region}(3 \mathrm{c})],}
\end{array}
$$

we obtain one de Sitter solution for $\tilde{B}_{(\mathrm{dS})}<0\left(B_{(\mathrm{dS})}>0\right.$, $\epsilon=-1$ ). In Table II] we show some examples (Models $\mathrm{C}, \mathrm{D}$, and $\mathrm{E})$. 


\begin{tabular}{|c|c|c|c|c|c|c|}
\hline model & $\left(c_{3}, c_{4}\right)$ & region & $\epsilon$ & $B_{\ell}$ & $\Lambda_{g} / m_{g}^{2}$ & vacuum \\
\hline \multirow[t]{4}{*}{$\mathrm{C}$} & \multirow[t]{4}{*}{$(1 / 2,0)$} & \multirow[t]{4}{*}{$(3 a)$} & -1 & $2+\sqrt{3}$ & $3 \sqrt{3}$ & $\mathrm{dS}$ \\
\hline & & & -1 & $2-\sqrt{3}$ & $-3 \sqrt{3}$ & $\mathrm{AdS}_{1}$ \\
\hline & & & 1 & 1 & 0 & $\mathrm{M}$ \\
\hline & & & 1 & 3 & -4 & $\mathrm{AdS}_{2}$ \\
\hline \multirow[t]{4}{*}{$\mathrm{D}$} & \multirow{4}{*}{$(5 / 2,-4)$} & \multirow[t]{4}{*}{$(3 b)$} & -1 & $2+\sqrt{3}$ & -72.3731 & $\mathrm{AdS}_{1}$ \\
\hline & & & -1 & $2-\sqrt{3}$ & 0.373067 & $\mathrm{dS}$ \\
\hline & & & 1 & $1 / 3$ & $-4 / 9$ & $\mathrm{AdS}_{2}$ \\
\hline & & & 1 & 1 & 0 & $\mathrm{M}$ \\
\hline \multirow[t]{4}{*}{$\bar{E}$} & \multirow[t]{4}{*}{$(3,0)$} & \multirow[t]{4}{*}{$(3 c)$} & -1 & 0.761557 & 29.7326 & $\mathrm{dS}$ \\
\hline & & & 1 & 0.636672 & -0.154054 & $\mathrm{AdS}_{1}$ \\
\hline & & & 1 & 1 & 0 & $\mathrm{M}$ \\
\hline & & & 1 & 4.12489 & -23.5786 & $\mathrm{AdS}_{2}$ \\
\hline
\end{tabular}

TABLE II: In the parameter region (3), there exists one de Sitter solution with $\tilde{B}_{(\mathrm{dS})}<0(\epsilon=-1)$. We also find three other vacuum solutions (two AdS solutions as well as a trivial Minkowski spacetime). The region (3) is divided into three sub-regions $((3 \mathrm{a}),(3 \mathrm{~b})$ and $(3 \mathrm{c}))$ depending on the properties of the solutions. We assume $\kappa_{f}=\kappa_{g}$.

In Fig 1, we show the regions (1), (2) and (3) where de Sitter solution exists are shown on the $c_{3}-c_{4}$ plane. For Models A-E with appropriate coupling parameters, we will discuss the dynamics of spacetime later. They are typical models in each region.

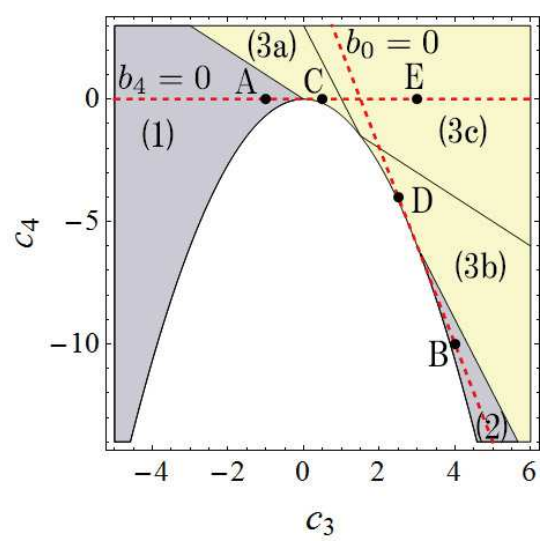

FIG. 1: The de Sitter solutions with $\epsilon=1$ and $\epsilon=-1$ are found in the regions (1) and (2) and in the region (3), respectively. The region (3) is divided into three sub-regions ((3a), (3b) and (3c)) by the dynamical behaviour of the universe.

The other two solutions $\left(\tilde{B}_{\left(\mathrm{AdS}_{1}\right)}\right.$ and $\left.\tilde{B}_{\left(\mathrm{AdS}_{2}\right)}\right)$, apart from the Minkowski spacetime, in the region (1)-(3) are anti de Sitter (AdS) spacetimes (see Tables पand I).

In the white region in Fig, 1, there is no de Sitter solution. There exist either three or one AdS spacetimes. We show some examples in Table III

\begin{tabular}{|c|c||c|c|c|c|c|}
\hline Model & $\left(c_{3}, c_{4}\right)$ & region & $\epsilon$ & $B$ & $\Lambda_{g} / m_{g}^{2}$ & vacuum \\
\hline \hline $\mathrm{F}$ & $(-2,-3)$ & \multirow{2}{*}{$(4)$} & -1 & 0.668907 & -53.3156 & $\mathrm{AdS}_{1}$ \\
\cline { 4 - 7 } & & & 1 & 1 & 0 & $\mathrm{M}$ \\
\cline { 4 - 7 } & & & 1 & 1.54181 & -0.221325 & $\mathrm{AdS}_{2}$ \\
\cline { 4 - 7 } & & & 1 & 2.3271 & -0.183049 & $\mathrm{AdS}_{3}$ \\
\hline \hline $\mathrm{G}$ & $(0,-1)$ & $(4)$ & -1 & 0.537656 & -15.3417 & $\mathrm{AdS}_{1}$ \\
\cline { 4 - 7 } & & & 1 & 1 & 0 & $\mathrm{M}$ \\
\hline
\end{tabular}

TABLE III: In the white region of Fig. 1 there is no de Sitter solution. We find only three AdS solutions or one AdS solution in addition to a trivial Minkowski spacetime. We assume $\kappa_{f}=\kappa_{g}$.

Note that the bare cosmological constant for $g_{\mu \nu}$ in the action is $b_{0}$, but the effective cosmological constant is given by $\Lambda_{g}$ through the interaction term. Hence, even if $b_{0} \leq 0$, as long as $\Lambda_{g}$ is positive, we find de Sitter spacetime as a vacuum solution.

\section{THE EVOLUTION OF THE UNIVERSE}

\section{A. Attractor Universes}

The matter and radiation densities become equal at the redshift $z=z_{\text {eq }} \approx 3000$ in our universe. Hence, after $z_{\text {eq }}$, matter density is dominant in $g$-spacetime, which we assume in what follows since we are interested in the present acceleration of the universe. We also assume a flat universe with $k=0$ from observation ${ }^{1}$.

We mainly discuss when radiation density in $f$ spacetime can be also ignored. In this case, (3.20) becomes

$$
C_{\Lambda}(\tilde{B}) a_{g}^{3}+C_{\mathrm{m}}(\tilde{B})=0
$$

which gives

$$
a_{g}(\tilde{B})=-\left(\frac{C_{\mathrm{m}}(\tilde{B})}{C_{\Lambda}(\tilde{B})}\right)^{\frac{1}{3}} .
$$

The potential for $\tilde{B}$ is given by

$V_{g}(\tilde{B})=-\frac{3 C_{\mathrm{m}} C_{\Lambda}^{2}\left[\kappa_{g}^{2} \rho_{g}^{[\gamma]} C_{\mathrm{m}}-c_{g, \mathrm{~m}} C_{\Lambda}+3 k C_{\Lambda}^{\frac{2}{3}} C_{\mathrm{m}}^{\frac{1}{3}}\right]}{\left(C_{\Lambda} C_{\mathrm{m}}^{\prime}-C_{\mathrm{m}} C_{\Lambda}^{\prime}\right)^{2}}$.

If $C_{\Lambda}(\tilde{B})=0$ as well as $C_{\mathrm{m}}(\tilde{B})=0$ initially, $\tilde{B}$ is always constant and then we find the homothetic solution as an exact solution:

$$
\begin{aligned}
& A=B=\left|\tilde{B}_{\ell}\right|, \\
& c_{f, \mathrm{~m}}=\left|\tilde{B}_{\ell}\right| c_{g, \mathrm{~m}}
\end{aligned}
$$

\footnotetext{
1 Even if we consider the closed $(k=1)$ or open $(k=-1)$ FLRW universe, our main result will not change.
} 


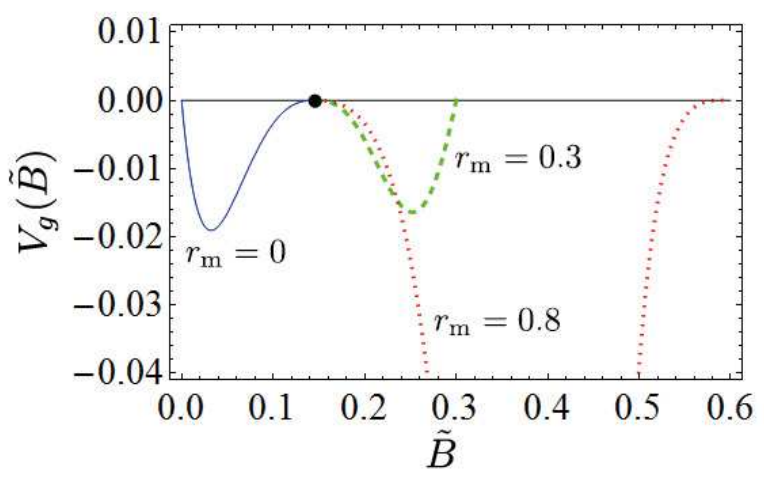

FIG. 2: The potentials $V_{g}(\tilde{B})$ for Model B $\left(c_{3}=4, c_{4}=-10\right)$ with $r_{\mathrm{m}}=0$ (the blue solid curve), $r_{\mathrm{m}}=0.3$ (the green dashed curve), or $r_{\mathrm{m}}=0.8$ (the red dotted curve). The black dot denotes de Sitter solution $\tilde{B}_{(\mathrm{dS})}$.

We find the conventional matter dominant universe with/without a cosmological constant 27, 35].

However, $C_{\Lambda}(\tilde{B})$ does not usually vanish. For generic initial data, solving the equation (3.25) for $\tilde{B}$, we obtain the scale factor $a_{g}$ by Eq. (4.2) with $\tilde{B}(t)$, and then another scale factor $a_{f}$ by

$$
a_{f}(\tilde{B})=\epsilon \tilde{B} a_{g}(\tilde{B}) .
$$

The ratio $A$ of the lapse functions is also given by

$$
A(\tilde{B})=\epsilon\left(\tilde{B}+\frac{3 C_{\mathrm{m}} C_{\Lambda}}{C_{\Lambda} C_{\mathrm{m}}^{\prime}-C_{\mathrm{m}} C_{\Lambda}^{\prime}}\right) .
$$

The potential $V_{g}$ satisfies the following conditions at $\tilde{B}=\tilde{B}_{\ell}$ :

$$
\begin{aligned}
V_{g}\left(\tilde{B}_{\ell}\right) & =0, \\
V_{g}^{\prime}\left(\tilde{B}_{\ell}\right) & =0, \\
V_{g}^{\prime \prime}\left(\tilde{B}_{\ell}\right) & =-6 \Lambda_{g}\left(\tilde{B}_{\ell}\right) .
\end{aligned}
$$

The AdS solution with $\Lambda_{g}<0$ is isolated because the potential is not negative definite and then Eq. (3.25) is satisfied only at $\tilde{B}_{\ell}=\tilde{B}_{(\mathrm{AdS})}$. For the case of $\Lambda_{g}>0$, on the other hand, there are two allowed regions where the universe can exist; the left and right regions of the point $\tilde{B}_{\ell}=\tilde{B}_{(\mathrm{dS})}$. The potential near $\tilde{B}_{(\mathrm{dS})}$ is shown in Fig. 2. The potential form depends on the ratio of matter densities $r_{\mathrm{m}} \equiv c_{f, \mathrm{~m}} / c_{g, \mathrm{~m}}$ as well as the coupling parameters $\left\{b_{i}\right\}$. Although there are two allowed regions in the equation of motion for $\tilde{B}$, one side is not physical, that is, it corresponds to the region where a scale factor becomes negative because from Eq. (4.2), we can evaluate the scale factor near $\tilde{B}=\tilde{B}_{(\mathrm{dS})}$ as

$$
\begin{aligned}
a_{g} & =-\left[\frac{c_{g, \mathrm{~m}} \tilde{B}_{(\mathrm{dS})}-\epsilon c_{f, \mathrm{~m}}}{C_{\Lambda}^{\prime}\left(\tilde{B}_{(\mathrm{dS})}\right)\left(\tilde{B}-\tilde{B}_{(\mathrm{dS})}\right)}\right]^{1 / 3} \\
& \propto\left(\tilde{B}-\tilde{B}_{(\mathrm{dS})}\right)^{-1 / 3}
\end{aligned}
$$

which changes the sign at $\tilde{B}=\tilde{B}_{(\mathrm{dS})}$. Here $C_{\Lambda}^{\prime}\left(\tilde{B}_{(\mathrm{dS})}\right)$ is a constant. Which side of regions is physical depends on the value of $r_{\mathrm{m}}$. For example, for Model B $\left(c_{3}=4\right.$ and $\left.c_{4}=-10\right)$, if $r_{\mathrm{m}}<r_{\mathrm{m}}^{(\mathrm{dS})}=0.145979$, the left region is physically allowed, while for $r_{\mathrm{m}}>r_{\mathrm{m}}^{(\mathrm{dS})}$, the right region becomes physically possible (see Fig. 2, in which we plot both cases of $r_{\mathrm{m}}=0$, and 0.3 ).

In both cases, $\tilde{B}$ evolves into $\tilde{B}_{(\mathrm{dS})}$ as an attractor. Near $\tilde{B}_{(\mathrm{dS})}$, the potential is approximated as

$$
V_{g} \approx-3 \Lambda_{g}\left(\tilde{B}-\tilde{B}_{(\mathrm{dS})}\right)^{2} .
$$

Hence we find the solution for $\tilde{B}$ as

$$
\tilde{B} \approx \tilde{B}_{(\mathrm{dS})}+C_{0} \exp \left[ \pm \sqrt{3 \Lambda_{g}} t\right],
$$

where $C_{0}$ is an integration constant. The plus sign corresponds to an unstable evolution rolling down from the potential peak, while the minus sign shows a stable solution which asymptotically approaches to $\tilde{B}_{(\mathrm{dS})}$. The scale factor evolves as

$$
a_{g} \propto \exp \left[\sqrt{\frac{\Lambda_{g}}{3}} t\right]
$$

(see Fig. 3). Hence de Sitter accelerating universe is obtained as an attractor. Note that if $r_{\mathrm{cr}}^{(\mathrm{dS})}<r_{\mathrm{m}}(<$ $\left.r_{\mathrm{m}}^{(\mathrm{AdS})}=1.67319\right)$, where $r_{\mathrm{cr}}^{(\mathrm{dS})}=0.41105$, the potential is unbounded from below and diverges at a finite value of $\tilde{B}$, where a singularity $(\dot{\tilde{B}}=\infty)$ appears as we will show later (see the potential with $r_{\mathrm{m}}=0.8$ in Fig. 2).

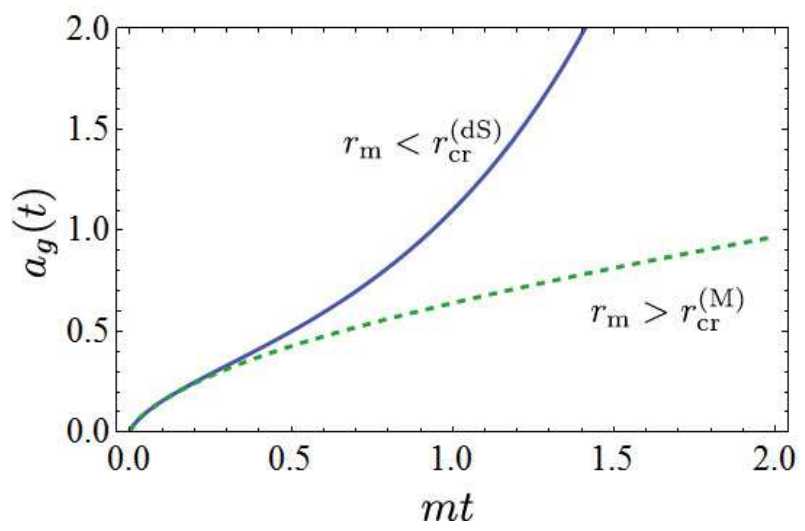

FIG. 3: The evolution of the scale factor $a_{g}$ for the case of $c_{3}=$ $4, c_{4}=-10$. The bottom curve corresponding to $c_{f, \mathrm{~m}} / c_{g, \mathrm{~m}}$ $=2$ (dashed green) shows the evolution to dust dominated universe, while the top curve corresponding to $c_{f, \mathrm{~m}} / c_{g, \mathrm{~m}}=0$ (solid blue) shows the evolution to de Sitter spacetime.

For the case of $\tilde{B}_{(\mathrm{M})}=1$, we find

$$
V_{g}\left(\tilde{B}_{(\mathrm{M})}\right)=V_{g}^{\prime}\left(\tilde{B}_{(\mathrm{M})}\right)=V_{g}^{\prime \prime}\left(\tilde{B}_{(\mathrm{M})}\right)=0,
$$


since $\Lambda_{g}=0$. Evaluating also $V_{g}^{\prime \prime \prime}\left(\tilde{B}_{(\mathrm{M})}\right)$ as

$$
V_{g}^{\prime \prime \prime}\left(\tilde{B}_{(\mathrm{M})}\right)=54\left(\frac{m_{f}^{2}+m_{g}^{2} r_{\mathrm{m}}}{1-r_{\mathrm{m}}}\right),
$$

we find that $V_{g}^{\prime \prime \prime}$ is positive when $r_{\mathrm{m}}<1$, then the left region $\left(\tilde{B} \leq \tilde{B}_{(\mathrm{M})}\right)$ is physically allowed, while the right region $\left(\tilde{B} \geq \tilde{B}_{(\mathrm{M})}\right)$ is allowed if $r_{\mathrm{m}}>1$.

In this case, the potential is approximated as

$$
V_{g}=V_{0}\left(\tilde{B}-\tilde{B}_{(\mathrm{M})}\right)^{3}
$$

with

$$
V_{0}=9\left(\frac{m_{f}^{2}+m_{g}^{2} r_{\mathrm{m}}}{1-r_{\mathrm{m}}}\right) .
$$

Eq. (3.25) is integrated as

$$
-V_{0}\left(\tilde{B}-\tilde{B}_{(\mathrm{M})}\right)=\frac{4}{\left(t-t_{0}\right)^{2}},
$$

where $t_{0}$ is an integration constant. As a result, the asymptotic solution of the scale factor is

$$
a_{g} \propto\left(\tilde{B}-\tilde{B}_{(\mathrm{M})}\right)^{-1 / 3} \propto\left(t-t_{0}\right)^{2 / 3},
$$

which is that of dust matter dominated universe (see Fig. 3). When $\tilde{B}_{\ell}=\tilde{B}_{(\mathrm{M})}$, a dust matter dominated universe is found as an attractor.

\section{B. Dynamics of the Universe with Twin Matter}

We are interested in whether the cosmic no-hair conjecture holds. Hence we analyze our system for various initial data and discuss which initial condition leads to de Sitter expansion. In order to discuss whether de Sitter accelerating universe is naturally achieved as an attractor or not, we survey all possible allowed initial data. Especially we focus on the ratio $r_{\mathrm{m}}$ of energy densities of twin matter fluids. The results are summarized on the $r_{\mathrm{m}}-\tilde{B}$ plane. For the parameter region (1) and (2), we show two typical examples of Model $\mathrm{A}\left(c_{3}=-1, c_{4}=0\right)$ and of Model B $\left(c_{3}=4, c_{4}=-10\right)$, in Figs. 4 and 5 , respectively. For the region (3), we also present the typical results for Model $\mathrm{C}\left(c_{3}=1 / 2, c_{4}=0\right), \mathrm{D}\left(c_{3}=5 / 2, c_{4}=-4\right)$ and $\mathrm{E}$ $\left(c_{3}=3, c_{4}=0\right)$ in Figs. 6 . 7 and 8 respectively.

The colored regions denote the ranges of physically allowed initial data. The universes in the stripe-shaded light-blue area evolve into de Sitter spacetime, while those in the crosshatched light-green area evolve into the dust matter dominated universe. The universes started from the grey shaded areas eventually find a future singularity.

We may probably easily understand that the spacetime evolves either de Sitter universe or the matter dominant

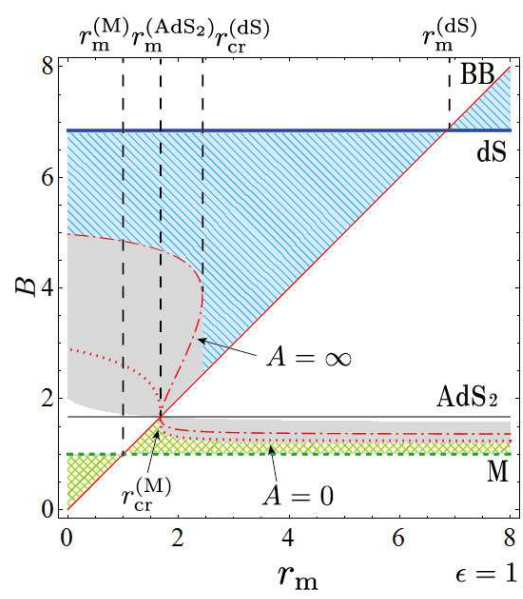

FIG. 4: The attractor regions in the $r_{\mathrm{m}}-\tilde{B}$ plane are shown for Model A $\left(c_{3}=-1, c_{4}=0\right)$. The solid, dotted and dashed lines denote de Sitter, anti de Sitter and dust dominated universes, respectively. $r_{\mathrm{m}}^{(\mathrm{M})}=1, r_{\mathrm{m}}^{\left(\mathrm{AdS}_{2}\right)}=1.67319$, and $r_{\mathrm{m}}^{(\mathrm{dS})}=6.85028$ give the boundary values, where the properties of dynamics change. The initial data in the striped-shaded light-blue and crosshatched light-green regions evolve into de Sitter and the dust dominated universe, respectively. BB denotes an initial Big Bang singularity $\left(a_{g}=a_{f}=0\right)$. The spacetime started from the other colored region evolves into singularities, which are shown by dot-dashed curves. There exist two critical values $r_{\mathrm{cr}}^{(\mathrm{dS})}=2.4328$ and $r_{\mathrm{cr}}^{(\mathrm{M})}=1.67318$. Beyond $r_{\mathrm{cr}}^{(\mathrm{dS})}$, every spacetime evolves into de Sitter universe if $B>r_{\mathrm{m}}^{\left(\mathrm{AdS}_{2}\right)}$, while all spacetime with $r_{\mathrm{m}}<r_{\mathrm{cr}}^{(\mathrm{M})}$ evolves into the matter dominant universe if $B<r_{\mathrm{m}}^{\left(\mathrm{AdS}_{2}\right)}$.

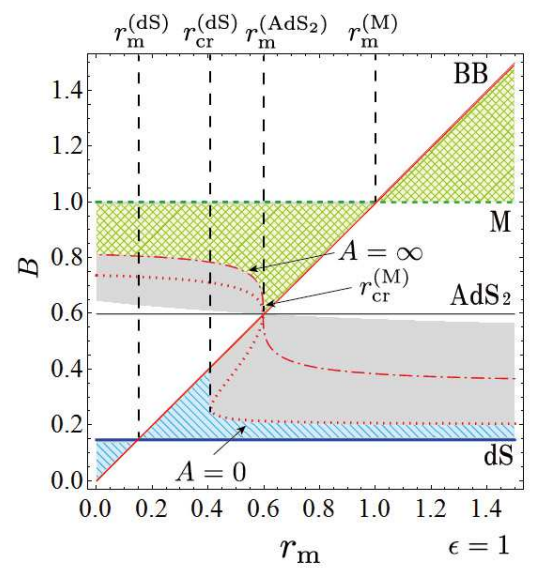

FIG. 5: The same figure as Fig. 4 for Model B $\left(c_{3}=\right.$ $\left.4, c_{4}=-10\right)$. The boundary values are given by $r_{\mathrm{m}}^{(\mathrm{M})}=1$, $r_{\mathrm{m}}^{\left(\mathrm{AdS}_{2}\right)}=0.59766$, and $r_{\mathrm{m}}^{(\mathrm{dS})}=0.145979$. Below the critical value $r_{\mathrm{cr}}^{(\mathrm{dS})}=0.41105$, every spacetime evolves into de Sitter universe if $B<r_{\mathrm{m}}^{\left(\mathrm{AdS}_{2}\right)}$, while all spacetime with $r_{\mathrm{m}}>r_{\mathrm{cr}}^{(\mathrm{M})}=0.597663$ evolves into the matter dominant universe if $B>r_{\mathrm{m}}^{\left(\mathrm{AdS}_{2}\right)}$. 

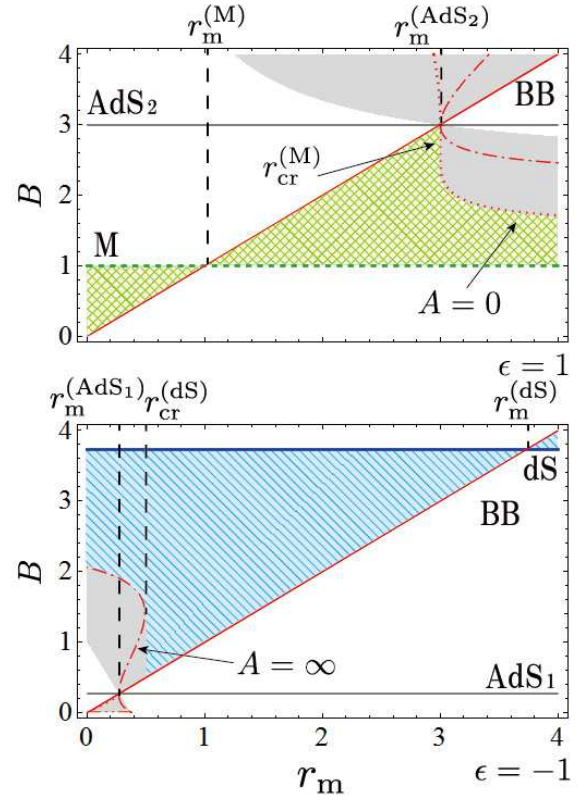

FIG. 6: The same figure as Fig. 4 for Model C $\left(c_{3}=1 / 2, c_{4}=\right.$ 0 ). The de Sitter solution exists in the case of $\epsilon=-1$, while the matter dominant universe is found for $\epsilon=1$. Hence we draw two figures of $\epsilon= \pm 1$ separately. because there appears a singularity at $\tilde{B}=0$, where $\epsilon$ changes the sign. The boundary values are given by $r_{\mathrm{m}}^{(\mathrm{M})}=1, r_{\mathrm{m}}^{\left(\mathrm{AdS}_{1}\right)}=2-\sqrt{3}$, $r_{\mathrm{m}}^{\left(\mathrm{AdS}_{2}\right)}=3$, and $r_{\mathrm{m}}^{(\mathrm{dS})}=2+\sqrt{3}$. Beyond the critical value $r_{\mathrm{cr}}^{(\mathrm{dS})}=0.489757$, every spacetime evolves into de Sitter universe if $\epsilon=-1$ and $B>r_{\mathrm{m}}^{\left(\mathrm{AdS}_{1}\right)}$, while all spacetime with $r_{\mathrm{m}}<r_{\mathrm{cr}}^{(\mathrm{M})}=2.99645$ evolves into the matter dominant universe if $\epsilon=1$ and $B<r_{\mathrm{m}}^{\left(\mathrm{AdS}_{2}\right)}$.

universe, depending on the initial conditions, because two homothetic solutions are attractors. However we also find singular spacetime for some initial data. Why a flat FLRW universe can evolves into a future singularity, which never happens in GR ? To explain how the universe evolves into a singularity, we consider Model B $\left(c_{3}=4\right.$ and $\left.c_{4}=-10\right)$ (Fig. 5). If $r_{\mathrm{m}}<r_{\mathrm{cr}}^{(\mathrm{dS})}$, the universe starts from a big bang initial singularity and evolves into de Sitter spacetime. When $r_{\mathrm{cr}}^{(\mathrm{dS})}<r_{\mathrm{m}}<r_{\mathrm{m}}^{\left(\mathrm{AdS}_{2}\right)}$, the evolution of $a_{g}$ is the similar to the above case. Starting from a big bang initial data $\left(a_{g}=0\right)$, it evolves into de Sitter spacetime. However the behaviour of $a_{f}$ becomes strange. We show the time evolution of two scale factors in Figs. 9, 11, in which we set $r_{\mathrm{m}}=0.58$.

$a_{f}$ first increases and then turns to decrease. It eventually increases again, resulting in an exponential expansion. In order to analyze the reason why the universe shows a transient collapse, we show the time evolution of $A$ and $B$ in Fig. 10. We find that $A$ becomes negative when $a_{f}$ decreases. It means that the time direction in this period turns to be reverse. It is the reason of the collapse.

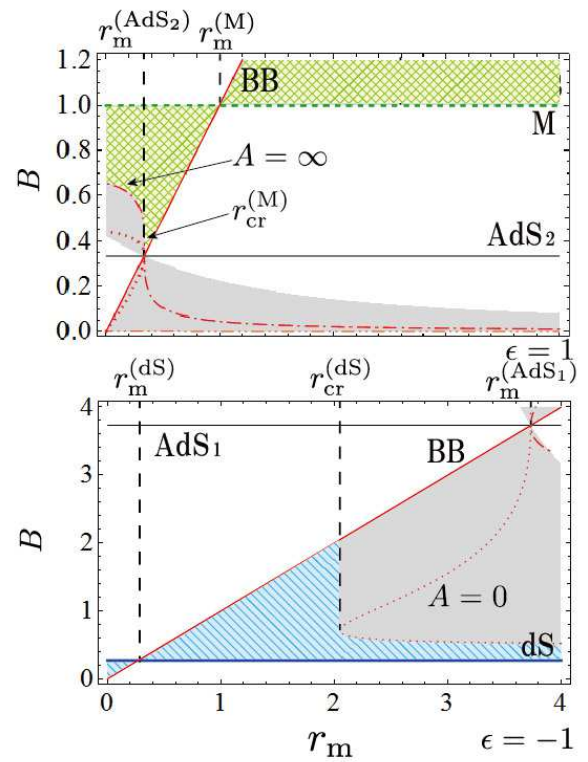

FIG. 7: The same figure as Fig. [6 for Model D $\left(c_{3}=5 / 2, c_{4}=\right.$ -4) $(\epsilon=-1)$. The boundary values are given by $r_{\mathrm{m}}^{(\mathrm{M})}=1$, $r_{\mathrm{m}}^{\left(\mathrm{AdS}_{1}\right)}=2+\sqrt{3}, r_{\mathrm{m}}^{\left(\mathrm{AdS}_{2}\right)}=1 / 3$, and $r_{\mathrm{m}}^{(\mathrm{dS})}=2-\sqrt{3}$. Below the critical value $r_{\mathrm{cr}}^{(\mathrm{dS})}=2.04183$, every spacetime evolves into de Sitter universe if $\epsilon=-1$ and $B<r_{\mathrm{m}}^{\left(\mathrm{AdS}_{1}\right)}$, while all spacetime with $r_{\mathrm{m}}>r_{\mathrm{cr}}^{(\mathrm{M})}=0.33729$ evolves into the matter dominant universe if $\epsilon=1$ and $B>r_{\mathrm{m}}^{\left(\mathrm{AdS}_{2}\right)}$.

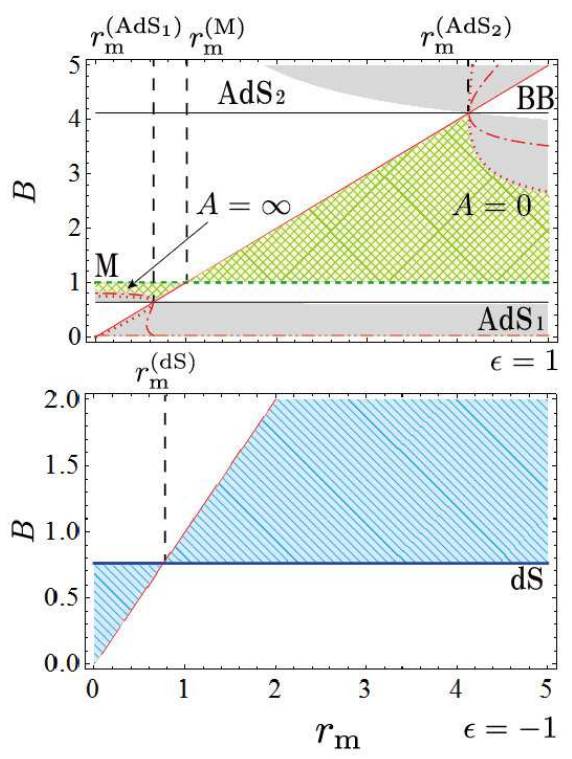

FIG. 8: The same figure as Fig. 6 for Model E $\left(c_{3}=3, c_{4}=\right.$ $0)$. The boundary values are given by $r_{\mathrm{m}}^{(\mathrm{M})}=1, r_{\mathrm{m}}^{\left(\mathrm{AdS}_{1}\right)}=$ $0.636672, r_{\mathrm{m}}^{\left(\mathrm{AdS}_{2}\right)}=4.12489$, and $r_{\mathrm{m}}^{(\mathrm{dS})}=0.761557$. Every spacetime with $\epsilon=-1$ evolves into de Sitter universe. The matter dominant universe is found for all spacetime with $\epsilon=1$ and $r_{\mathrm{m}}^{\left(\mathrm{AdS}_{1}\right)}<r_{\mathrm{m}}<r_{\mathrm{m}}^{\left(\mathrm{AdS}_{2}\right)}$ 


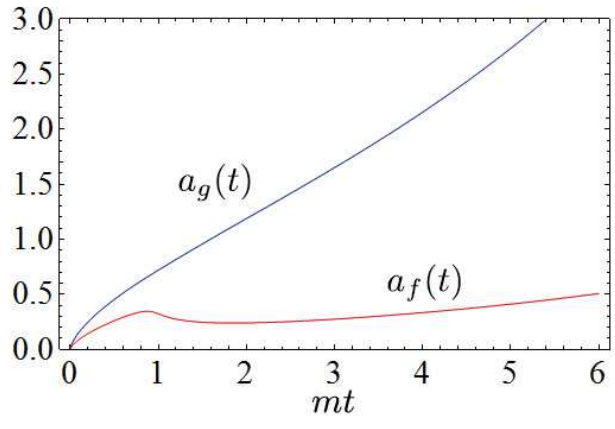

FIG. 9: The time evolution of two scale factors $a_{g}$ and $a_{f}$ for Model B $\left(c_{3}=4, c_{4}=-10\right)$ with $c_{f, \mathrm{~m}} / c_{g, \mathrm{~m}}=0.58$.

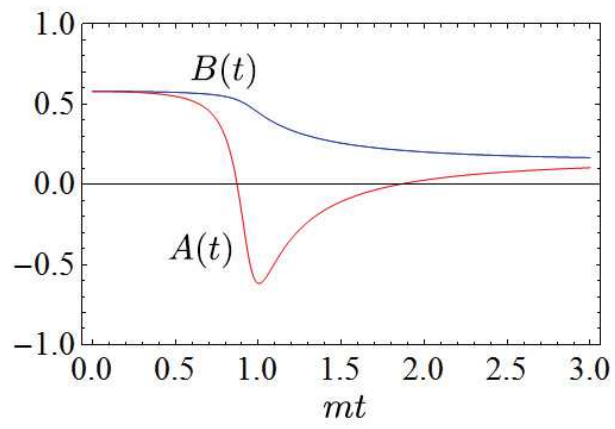

FIG. 10: The time evolution of $B(t)$ and $A(t)$ for Model B with $c_{f, \mathrm{~m}} / c_{g, \mathrm{~m}}=0.58$.

However there appears a singularity when $\tilde{A}$ vanishes, i.e., $\dot{a}_{f}=0$. Substituting $\tilde{A}=\dot{a}_{f} / \dot{a}_{g}$ into the Ricci scalar $\mathcal{R}(f)$, we obtain

$$
\begin{aligned}
\mathcal{R}(f) & =6\left[\frac{1}{N_{f} a_{f}}\left(\frac{\dot{a}_{f}}{N_{f}}\right)+\frac{\dot{a}_{f}^{2}}{N_{f}^{2} a_{f}^{2}}+\frac{k}{a_{f}^{2}}\right] \\
& =6\left(\frac{\dot{a}_{g} \ddot{a}_{g}}{a_{f} \dot{a}_{f}}+\frac{\dot{a}_{g}^{2}}{a_{f}^{2}}+\frac{k}{a_{f}^{2}}\right) .
\end{aligned}
$$

$\ddot{a_{g}}$ does not vanish at $\tilde{A}=0$, because the $r-r$ component of field equation of $g_{\mu \nu}$ is given by

$$
\begin{gathered}
2 \frac{\ddot{a_{g}}}{a_{g}}+\frac{\dot{a_{g}}}{a_{g}^{2}}+\frac{k}{a_{g}^{2}}=m_{g}^{2}\left[b_{0}+b_{1}(\tilde{A}+2 \tilde{B})\right. \\
\left.+b_{2}\left(2 \tilde{A} \tilde{B}+\tilde{B}^{2}\right)+b_{3} \tilde{A} \tilde{B}^{2}\right]-\kappa_{g}^{2} P_{g} .
\end{gathered}
$$

Thus, the Ricci scalar $\mathcal{R}(f)$ diverges at $\dot{a}_{f}=0(\tilde{A}=0)$ assuming $\dot{a}_{g} \neq 0$. Note that the Ricci scalar for $g_{\mu \nu}$ is finite at this point. It implies that $g$-spacetime is regular whereas $f$-spacetime is singular at $\tilde{A}=0$.

Note that it is possible to solve the equation for $\tilde{B}$ by use of the potential $V_{g}$ even we find a singularity in $f$ spacetime, because the interaction term does not diverge.
However, it is impossible to solve the equation by use of the cosmic time $\tau_{f}$ in $f$-spacetime. From Fig. 11, it is

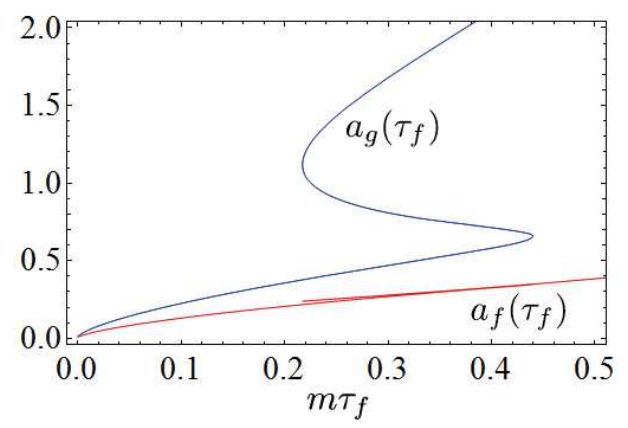

FIG. 11: The time evolution of two scale factors $a_{g}$ and $a_{f}$ in terms of $\tau_{f}$ for Model B with $c_{f, \mathrm{~m}} / c_{g, \mathrm{~m}}=0.58$. Beyond the singularity, both scale factors decrease in time, and then increase again after another singularity.

almost trivial that a singularity appears at the turning points of $a_{f}$.

Fig 10 implies that $t\left(\tau_{f}\right)$ is not single-valued although $\tau_{f}(t)$ is a single-valued function. As a result, the variables such as $a_{g}\left(\tau_{f}\right)$ or $a_{f}\left(\tau_{f}\right)$ are not single-valued (see Fig.11).

Beyond a singularity, however, since there is no natural junction condition at the singularity, we can change the sign of the lapse function $N_{f}$, which is negative when $A$ is negative. Since the tetrad in $f$-spacetime is given by (3.4), if we change the sign of $N_{f}$, we also have to change the sign of the spatial part. Because the scale factor $a_{f}$ must be positive, we have to reverse the spatial direction, that is, we should change the parity in $f$-spacetime. Hence if we keep the time direction in $f$-spacetime beyond a singularity, we have to change the parity for the period of $A<0$. Although there is no contraction phase in $f$-spacetime as well as in $g$-spacetime, the scale factor in $f$-metric $a_{f}$ becomes discontinuous (see Fig. 12).

The reverse of the above case also occurs, that is, $f$ spacetime is regular anytime except for a big bang singularity whereas $g$-spacetime becomes singular at $\dot{a}_{g}=0$, when $\tilde{A}=\infty$. The sign of $\tilde{A}$ also changes beyond this singularity. This happens in the case of Model A $\left(c_{3}=-1, c_{4}=0\right)$.

In Figs. 4,8, we show the region of $\tilde{A}<0$, on which boundaries (the solid and dashed curves for $\tilde{A}=0$ $\tilde{A}=\infty$, respectively) singularities appear. Hence if the universe starts from the gray shaded area, it evolves into a singularity either at $\tilde{A}=0$ or at $\tilde{A}=\infty$. If the universe starts from a big bang singularity $\left(a_{g}=a_{f}=0\right.$ : red solid line), it evolves into a negative lapse area through a singularity and eventually goes to a positive lapse area again, finding de Sitter accelerating universe (or the matter dominated universe). For the other initial data in the grey area, the boundary does not correspond to a 


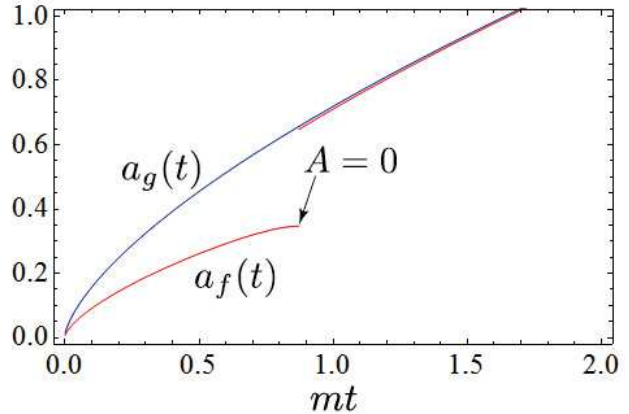

FIG. 12: The time evolution of two scale factors for the same parameters as those in Fig. 9] Although both scale factors increase in time, there still exists a singularity at $A=0\left(\dot{a}_{f}=\right.$ $0)$ and $a_{f}$ is discontinuous there. The parity of $f$-spacetime is changed beyond the singularity.

big bang singularity, but the universe is bounced at the boundary. Either this spacetime evolves directly into a singularity at $\tilde{A}=\infty$, or it first goes to the boundary and then it is bounced back to the singularity. Going through a negative lapse area, both cases eventually evolve into a positive lapse area again. In any case, however, a singularity formation cannot be avoided if the universe starts from the grey area.

As shown in Figs. 4, there exists critical values $r_{\mathrm{cr}}^{(\mathrm{dS})}\left(r_{\mathrm{cr}}^{(\mathrm{M})}\right)$ for $r_{\mathrm{m}}=c_{f, \mathrm{~m}} / c_{g, \mathrm{~m}}$, beyond (or below) which both $g$ - and $f$-spacetime are regular and then they evolve into de Sitter universe (or the dust matter dominated universe). The universe never evolves into a singularity.

The critical value $r_{\mathrm{cr}}^{(\mathrm{dS} / \mathrm{M})}$ can be found as follows: It is given by an extreme value of boundary curve of $\tilde{A}=0$ or $\tilde{A}=\infty$, which are given by

$$
\begin{aligned}
& \left.r_{\mathrm{m}}\right|_{A=0}=\left.\frac{c_{f, \mathrm{~m}}}{c_{g, \mathrm{~m}}}\right|_{A=0}=\epsilon \tilde{B}\left(1+\frac{C_{\Lambda}}{3 C_{\Lambda}-\tilde{B} C_{\Lambda}^{\prime}}\right) \\
& \left.r_{\mathrm{m}}\right|_{A=\infty}=\left.\frac{c_{f, \mathrm{~m}}}{c_{g, \mathrm{~m}}}\right|_{A=\infty}=\epsilon\left(\tilde{B}-\frac{C_{\Lambda}}{C_{\Lambda}^{\prime}}\right) .
\end{aligned}
$$

The extremal condition gives the equation for $\tilde{B}$ at the critical point such that

$$
\left(\kappa_{g}^{2} b_{1}-\kappa_{f}^{2} b_{3}\right) \tilde{B}^{2}+\left(\kappa_{g}^{2} b_{0}-3 \kappa_{f}^{2} b_{2}\right) \tilde{B}-2 \kappa_{f}^{2} b_{1}=0,
$$

for $\tilde{A}=0$ or

$$
2 \kappa_{g}^{2} b_{3} \tilde{B}^{2}+\left(3 \kappa_{g}^{2} b_{2}-\kappa_{f}^{2} b_{4}\right) \tilde{B}+\left(\kappa_{g}^{2} b_{1}-\kappa_{f}^{2} b_{3}\right)=0
$$

for $\tilde{A}=\infty$, respectively. The roots of the above equation just provide a candidate for the critical value $\tilde{B}_{\text {cr }}$. Since the critical point must exist in the physically allowed region, we have to impose the additional constraint for the critical value as

$$
V_{g}\left(\tilde{B}_{\mathrm{cr}}, r_{\mathrm{cr}}^{(\mathrm{dS} / \mathrm{M})}\right)<0 .
$$

for $\tilde{B}_{(\mathrm{dS} / \mathrm{M})}>\tilde{B}_{\mathrm{cr}}>r_{\mathrm{m}}$ or $\tilde{B}_{(\mathrm{dS} / \mathrm{M})}<\tilde{B}_{\mathrm{cr}}<r_{\mathrm{m}}$. These critical values $r_{\mathrm{cr}}^{(\mathrm{dS} / \mathrm{M})}$ are shown in Figs. 4,8.

We summarize the results in this subsection in Table IV.

\begin{tabular}{|c||c|c|c|}
\hline region & $\epsilon$ & $r_{\mathrm{m}}$ & $B$ \\
\hline \hline \multicolumn{4}{|c|}{ de Sitter accelerating universe } \\
\hline$(1)$ & 1 & $r_{\mathrm{m}}>r_{\mathrm{cr}}^{(\mathrm{dS})}$ & $B>r_{\mathrm{m}}^{\left(\mathrm{AdS}_{2}\right)}$ \\
\hline$(2)$ & 1 & $r_{\mathrm{m}}<r_{\mathrm{cr}}^{(\mathrm{dS})}$ & $B<r_{\mathrm{m}}^{\left(\mathrm{AdS}_{2}\right)}$ \\
\hline$(3 \mathrm{a})$ & -1 & $r_{\mathrm{m}}>r_{\mathrm{cr}}^{(\mathrm{dS})}$ & $B>r_{\mathrm{m}}^{\left(\mathrm{AdS}_{1}\right)}$ \\
\hline$(3 \mathrm{~b})$ & -1 & $r_{\mathrm{m}}<r_{\mathrm{cr}}^{(\mathrm{dS})}$ & $B<r_{\mathrm{m}}^{\left(\mathrm{AdS}_{1}\right)}$ \\
\hline$(3 \mathrm{c})$ & -1 & no condition & no condition \\
\hline \hline \multicolumn{4}{|c|}{ matter dominant universe } \\
\hline$(1)$ & 1 & $r_{\mathrm{m}}<r_{\mathrm{cr}}^{(\mathrm{M})}$ & $B<r_{\mathrm{m}}^{\left(\mathrm{AdS}_{2}\right)}$ \\
\hline$(2)$ & 1 & $r_{\mathrm{m}}>r_{\mathrm{cr}}^{(\mathrm{M})}$ & $B>r_{\mathrm{m}}^{\left(\mathrm{AdS}_{2}\right)}$ \\
\hline$(3 \mathrm{a})$ & 1 & $r_{\mathrm{m}}<r_{\mathrm{cr}}^{(\mathrm{M})}$ & $B<r_{\mathrm{m}}^{\left(\mathrm{AdS}_{2}\right)}$ \\
\hline$(3 \mathrm{~b})$ & 1 & $r_{\mathrm{m}}>r_{\mathrm{cr}}^{(\mathrm{M})}$ & $B>r_{\mathrm{m}}^{\left(\mathrm{AdS}_{2}\right)}$ \\
\hline$(3 \mathrm{c})$ & 1 & $r_{\mathrm{cr}}^{\left(\mathrm{AdS}_{1}\right)}<r_{\mathrm{m}}<r_{\mathrm{cr}}^{\left(\text {AdS }_{2}\right)}$ & $r_{\mathrm{cr}}^{\left(\text {AdS }_{1}\right)}<B<r_{\mathrm{cr}}^{\left(\mathrm{AdS}_{2}\right)}$ \\
\hline
\end{tabular}

TABLE IV: The conditions for de Sitter accelerating universe or the matter dominant universe. Every spacetime evolves into de Sitter universe or the matter dominant universe, if the given conditions are satisfied for $\epsilon, r_{\mathrm{m}}$ and the initial value of $B$.

We show the conditions for the ratio $r_{\mathrm{m}}$ and the initial value of $B$ under which every spacetime evolves into de Sitter universe or the matter dominant universe. The critical values depend on the coupling constants $\left\{b_{i}\right\}$ (or $c_{3}$ and $c_{4}$ ) and $\kappa_{f}^{2} / \kappa_{g}^{2}$. When spacetimes do not satisfy these conditions, the universe will find a singularity unless we fine-tune the initial conditions.

\section{Cosmic No Hair Conjecture}

In the previous subsection, we discuss several examples, in which we showed that there are three possibilities for the fate of spacetime: de Sitter accelerating universe, the matter dominant universe, and spacetime with a future singularity, depending on the initial condition. Hence in the exact sense, the cosmic no hair conjecture does not hold, but de Sitter universe can be obtained from a wide range of initial conditions. In this subsection, we shall further analyze how this result is generic by surveying the possible coupling parameters $\left\{b_{k}\right\}$, which are given by two free parameters $c_{3}$ and $c_{4}$ as (2.16).

Here, just for simplicity, we study two typical cases with one free parameter $c_{3}$ : (I) $b_{4}=c_{4}=0$ and (II) $b_{0}=4 c_{3}+c_{4}-6=0$. The first and second cases include the region (1), (3a) and (3c), and the region $(2),(3 \mathrm{~b})$ and $(3 \mathrm{c})$, respectively. (See the corresponding red dashed lines in Fig.1])

In Figs. 13 (a) and (b), for those two cases (I) and (II), we show which range of $r_{\mathrm{m}}$ can reach to de Sitter 


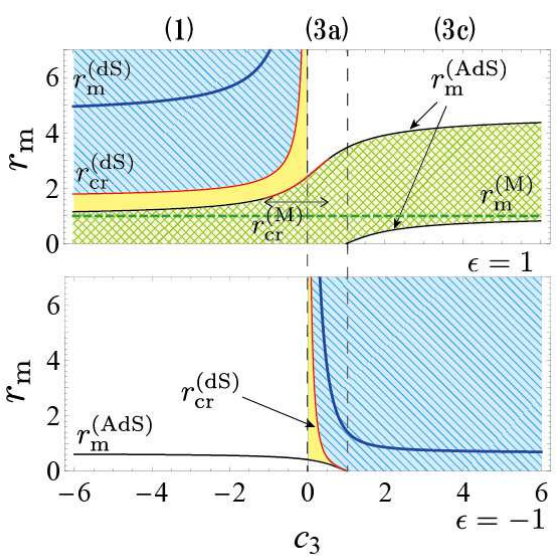

(a) Case (I)

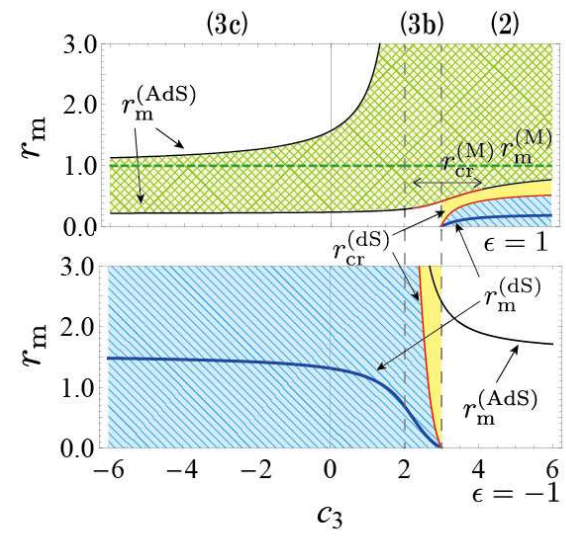

(b) Case (II)

FIG. 13: The de Sitter solution (blue curves) and the necessary condition of $r_{\mathrm{m}}$ for self-acceleration (the stripe-shaded light-blue regions) are shown. We also shown the matter dominate universe (the crosshatched light-green curve) and its necessary condition of $r_{\mathrm{m}}$. The dashed red curves show AdS solutions with $\Lambda_{g}<0$. If the universe starts from the yellow region, it evolves into a singularity. The critical value $r_{\mathrm{cr}}^{(\mathrm{dS})}$ exists in the regions (1), (2), (3a) and (3b). The another critical value $r_{\mathrm{cr}}^{(\mathrm{M})}$ appear if $-1.09<c_{3}<0.55$ for Case (I) and $2.27<c_{3}<4.09$ for Case (II), respectively.

universe or the matter dominant universe, The blue solid curve and green dashed line denote de Sitter solution and the matter dominated universe, respectively. For the dS solutions, the value of $\epsilon$ is negative in the regions (3a), $(3 \mathrm{~b})$ and $(3 \mathrm{c})$ while it is positive in the regions (1) and (2).

In the region (3c), all spacetime evolves into either de Sitter self-accelerating universe or matter dominant universe, except for the time reversed ones, which collapse into a big crunch. On the other hand, in the regions (1), (2), (3a) and (3b), there exists a critical value $r_{\mathrm{cr}}^{(\mathrm{dS})}$, beyond (below) which spacetime with an appropriate initial condition evolves into de Sitter universe.
However the case with other initial data will evolve into either matter dominant universe or find a singularity. We note the critical values $r_{\mathrm{cr}}^{(\mathrm{M})}$ are extremely close to $r_{\mathrm{m}}^{\text {(AdS) }}$ and these appear only if $-1.09<c_{3}<0.55$ for Case (I) and $2.27<c_{3}<4.09$ for Case (II). Outside these regions, magnitude relation between $B_{\mathrm{cr}}$ and $r_{\mathrm{m}}$ is $B_{\mathrm{cr}}>r_{\mathrm{m}}>r_{\mathrm{m}}^{(\mathrm{M})}$ or $B_{\mathrm{cr}}<r_{\mathrm{m}}<r_{\mathrm{m}}^{(\mathrm{M})}$, which dose not satisfy the additional constraint (4.24).

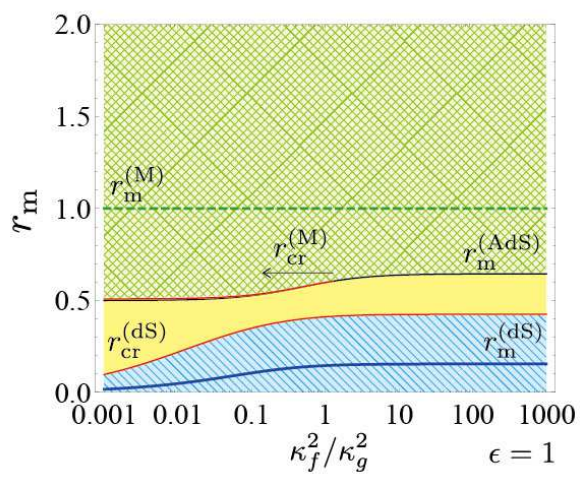

(a)

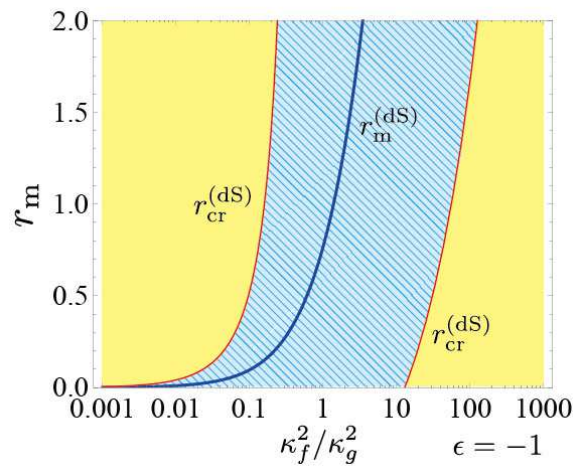

(b)

FIG. 14: The similar figures to Fig. 13 for different values of $\kappa_{f}^{2} / \kappa_{g}^{2}$. We plot the results for (a) Model B $\left(c_{3}=4, c_{4}=-10\right)$ and (b) Model E $\left(c_{3}=3, c_{4}=0\right)$. For Model B, in addition to the critical value $r_{\mathrm{cr}}^{(\mathrm{dS})}$ another critical value $r_{\mathrm{cr}}^{(\mathrm{M})}$ appears if $\kappa_{f}^{2} / \kappa_{g}^{2}<1.26$. For Model E, no critical value appears if $0.522408<\kappa_{f}^{2} / \kappa_{g}^{2}<13.0711$. All spacetime approach de Sitter universe.

In order to see the dependence of gravitational constants $\kappa_{g}$ and $\kappa_{f}$, we change the ratio of gravitational constants $\kappa_{f} / \kappa_{g}$ by fixing the coupling constants $\left\{b_{i}\right\}$. In Figs 14 (a) and (b), we show the results for different values of the ratio $\kappa_{g}^{2} / \kappa_{g}^{2}$ for Model B $\left(c_{3}=4, c_{4}=-10\right)$ and Model $\mathrm{E}\left(c_{3}=3, c_{4}=0\right)$, respectively.

The result is qualitatively same in Model B except for the existence of $r_{\mathrm{cr}}^{(\mathrm{M})}$, while it is quite different in Model E. For Model B, the critical value $r_{\mathrm{cr}}^{(\mathrm{M})}$ appears only if $\kappa_{f}^{2} / \kappa_{g}^{2}<1.26$ as shown in Fig. 13. For Model E, all spacetime evolves into de Sitter universe if $0.522408<$ 
$\kappa_{f}^{2} / \kappa_{g}^{2}<13.0711$, otherwise a critical value appears as Model B.

Hence we can conclude that no hair conjecture does not always hold in the exact sense, but a self-accelerating universe can be found from natural (not fine-tuned) initial data for general coupling parameters and gravitational constants.

We should note the effect of radiation. Although the present radiation density is much less than matter density in our universe, it may not be the case for $f$-matter fields. Analyzing the case that $f$-radiation density is not ignorable, we find that the dynamics of the universe does not change so much from the matter dominated case, although the interpretation of "dark matter" component will be different (see $\S$. VD).

\section{TOWARD THE $\Lambda$-CDM UNIVERSE}

\section{A. Effective Friedmann Equation}

As we show, de Sitter accelerating universe is realized for generic initial conditions in bi-gravity theory. One may wonder whether our present observed universe is found in this model. Among many cosmological models, the $\Lambda$-CDM model is most preferable from the observational view point [1]. The amount of cold dark matter is five times as large as the baryonic matter. Can we obtain such a model in the present theory as an attractor or without any fine-tuning ?

In order to study it, assuming the spacetime approaches the homothetic solution $\left(\tilde{B}=\tilde{B}_{\ell}\right)$, we describe our basic equation (3.15) in the form of a standard Friedmann equation. Since de Sitter spacetime or the matter dominant universe is an attractor in the present model, $\tilde{B}_{\ell}=\tilde{B}_{(\mathrm{dS})}$ or $\tilde{B}_{(\mathrm{M})}$.

We rewrite the interaction term $\rho_{g}^{[\gamma]}$ in terms of the energy densities of twin matter fluids, $\rho_{g}$ and $\rho_{f}$. Near $\tilde{B}=\tilde{B}_{\ell}$, this term is expanded as

$$
\kappa_{g}^{2} \rho_{g}^{[\gamma]} \approx \Lambda_{g}+R_{1}\left(\tilde{B}-\tilde{B}_{\ell}\right)+O\left(\left(\tilde{B}-\tilde{B}_{\ell}\right)^{2}\right),
$$

where

$$
R_{1} \equiv\left(\kappa_{g}^{2} \rho_{g}^{[\gamma]}\right)^{\prime}\left(\tilde{B}_{\ell}\right)=3 m_{g}^{2}\left(b_{1}+2 b_{2} \tilde{B}_{\ell}+b_{3} \tilde{B}_{\ell}^{2}\right)
$$

To evaluate $\left(\tilde{B}-\tilde{B}_{\ell}\right)$ in terms of matter densities, we expand Eq. (3.21) as

$$
\tilde{B}_{\ell} C_{\mathrm{m}}\left(\tilde{B}_{\ell}\right) a_{g}+C_{\mathrm{r}}\left(\tilde{B}_{\ell}\right)+\left[\tilde{B}_{\ell} C_{\Lambda}^{\prime}\left(\tilde{B}_{\ell}\right) a_{g}^{4}+\left[C_{\mathrm{m}}\left(\tilde{B}_{\ell}\right)+\tilde{B}_{\ell} C_{\mathrm{m}}^{\prime}\left(\tilde{B}_{\ell}\right)\right] a_{g}+C_{\mathrm{r}}^{\prime}\left(\tilde{B}_{\ell}\right)\right]\left(\tilde{B}-\tilde{B}_{\ell}\right)+O\left(\left(\tilde{B}-\tilde{B}_{\ell}\right)^{2}\right)=0
$$

where we use $C_{\Lambda}\left(\tilde{B}_{\ell}\right)=0$. In the limit of $\tilde{B} \rightarrow \tilde{B}_{\ell}$, if the universe is expanding, dropping the higher-order terms of $a_{g}^{-1}$ because $a_{g}$ is increasing, we find

$$
\tilde{B}-\tilde{B}_{\ell} \approx-\frac{1}{C_{\Lambda}^{\prime}\left(\tilde{B}_{\ell}\right)}\left[\frac{C_{\mathrm{m}}\left(\tilde{B}_{\ell}\right)}{a_{g}^{3}}+\frac{C_{\mathrm{r}}\left(\tilde{B}_{\ell}\right)}{\tilde{B}_{\ell} a_{g}^{4}}\right]+O\left(\frac{1}{a_{g}^{6}}\right) .
$$

Note that $\left(\tilde{B}-\tilde{B}_{\ell}\right) \sim O\left(a_{g}^{-3}\right)$. Plugging (5.3) into (5.1), we find

$$
\kappa_{g}^{2}\left(\rho_{g}^{[\gamma]}+\rho_{g}\right) \approx \Lambda_{g}+\kappa_{g}^{2} \rho_{g}^{(\mathrm{m})}\left[1-\frac{\left(1-\frac{\epsilon r_{\mathrm{m}}}{\tilde{B}_{\ell}}\right)}{\left(1-\frac{2 \Lambda_{g}}{3 m_{\text {eff }}^{2}}\right)\left(1+\frac{\kappa_{f}^{2}}{\tilde{B}_{\ell}^{2} \kappa_{g}^{2}}\right)}\right]+\kappa_{g}^{2} \rho_{g}^{(\mathrm{r})}\left[1-\frac{\left(1-\frac{r_{\mathrm{r}}}{\tilde{B}_{\ell}^{2}}\right)}{\left(1-\frac{2 \Lambda_{g}}{3 m_{\text {eff }}^{2}}\right)\left(1+\frac{\kappa_{f}^{2}}{\tilde{B}_{\ell}^{2} \kappa_{g}^{2}}\right)}\right]+O\left(\frac{1}{a_{g}^{6}}\right)
$$

where $\rho_{g,(\mathrm{~m})}$ and $\rho_{g,(\mathrm{r})}$ are energy densities of $g$-matter and $g$-radiation, respectively, and $r_{\mathrm{r}}=c_{f, \mathrm{r}} / c_{g, \mathrm{r}} \cdot m_{\mathrm{eff}}$ is the graviton mass in the present background spacetime, which is defined by Eq. (2.33).

Since the energy density of $f$-matter fluids in the present limit is approximated by

$$
\begin{aligned}
\kappa_{f}^{2} \rho_{f}= & \frac{c_{f, \mathrm{~m}}}{a_{f}^{3}}+\frac{c_{f, \mathrm{r}}}{a_{f}^{4}}=\frac{r_{\mathrm{m}}}{\tilde{B}^{3}} \frac{c_{g, \mathrm{~m}}}{a_{g}^{3}}+\frac{r_{\mathrm{m}}}{\tilde{B}^{4}} \frac{c_{g, \mathrm{r}}}{a_{g}^{4}} \\
& \approx \frac{r_{\mathrm{m}}}{\tilde{B}_{\ell}^{3}} \frac{c_{g, \mathrm{~m}}}{a_{g}^{3}}+\frac{r_{\mathrm{r}}}{\tilde{B}_{\ell}^{4}} \frac{c_{g, \mathrm{r}}}{a_{g}^{4}}+O\left(a_{g}^{-6}\right),
\end{aligned}
$$

replacing $r_{\mathrm{m}}$ and $r_{\mathrm{r}}$ by $f$-matter fluids, we finally obtain a standard form of the effective Friedmann equation in the present model as 
where

$$
\begin{aligned}
\kappa_{\text {eff }}^{2} & =\kappa_{g}^{2}\left[1-\frac{1}{\left(1-\frac{2 \Lambda_{g}}{3 m_{\text {eff }}^{2}}\right)\left(1+\frac{\kappa_{f}^{2}}{\tilde{B}_{\ell}^{2} \kappa_{g}^{2}}\right)}\right] \\
\rho_{\mathrm{D}} & =\frac{\kappa_{f}^{2} \tilde{B}_{\ell}^{2}}{\kappa_{g}^{2}\left[\left(1-\frac{2 \Lambda_{g}}{3 m_{\text {eff }}^{2}}\right)\left(1+\frac{\kappa_{f}^{2}}{\tilde{B}_{\ell}^{2} \kappa_{g}^{2}}\right)-1\right]} \rho_{f} .
\end{aligned}
$$

$\kappa_{\text {eff }}^{2}$ is the effective gravitational constant, which is always smaller than the bare gravitational constant $\kappa_{g^{2}}$, if the Higuchi bound is satisfied $\left(m_{\mathrm{eff}}^{2}>2 \Lambda_{g} / 3\right) . \rho_{\mathrm{D}}$ is regarded as "dark sector" which origin is another one of twin matter. In particular, when dust matter fluids are dominant, $\rho_{\mathrm{D}}$ is regarded as "dark matter".

Although the effective Friedmann equation (5.6) is valid both for an asymptotic de Sitter universe $\left(\tilde{B}_{\ell}=\right.$ $\left.\tilde{B}_{(\mathrm{dS})}\right)$ and for an asymptotic matter dominant universe $\left(\tilde{B}_{\ell}=\tilde{B}_{(\mathrm{M})}=1\right)$, in what follows, we discuss only the case of $\tilde{B}_{(\mathrm{dS})}$ to explain the present observed universe.

\section{B. Effective Gravitational Constant}

First of all, we discuss about the effective gravitational constant $\kappa_{\text {eff }}^{2}$, which must be positive in order for gravitational force to be attractive. In Table $\mathrm{V}$ we summarize the value of $\kappa_{\text {eff }}^{2}$ as well as $m_{\text {eff }}^{2}$ for five models (Models $\mathrm{A} \sim \mathrm{E})$, where we assume $\kappa_{f}^{2}=\kappa_{g}^{2}$. From Table $\mathrm{V}$, we can reject two models (Models $\mathrm{A}$ and $\mathrm{C}$ ) because those models predict a negative gravitational constant.

\begin{tabular}{|c||r|r|}
\hline Model & $\kappa_{\text {eff }}^{2} / \kappa_{g}^{2}$ & $m_{\text {eff }} / m$ \\
\hline A & -0.0880972 & 6.43151 \\
\hline $\mathrm{B}$ & 0.976813 & 0.938869 \\
\hline $\mathrm{C}$ & -0.108741 & 3.30578 \\
\hline $\mathrm{D}$ & 0.920396 & 0.885782 \\
\hline $\mathrm{E}$ & 0.0283764 & 3.99107 \\
\hline
\end{tabular}

TABLE V: The effective gravitational constant and the effective graviton mass in de Sitter background for Models A E. We assume $\kappa_{f}^{2}=\kappa_{g}^{2}$.

To see more general cases, we calculate the effective gravitational constant $\kappa_{\text {eff }}^{2}$ for two one-parameter families ((I) $b_{0}=0$ and (II) $b_{4}=0$ ), which we discussed in $\S$. IVC] Fig. 15] shows $\kappa_{\text {eff }}^{2}$ with respect to $c_{3}$. We find the constraint on $c_{3}$ as $c_{3}>1.67845$ for the case (I), while $c_{3}>2.61963$ for the case (II).

For most general parameters, in Fig. 16, we also show the range of $\kappa_{\text {eff }}^{2}>0$ by the stripe-shaded light-blue region in the $c_{3}-c_{4}$ plane.

If we change the ratio of $\kappa_{f}^{2} / \kappa_{g}^{2}$, the critical curve for $c_{3}$ and $c_{4}$ moves. We show the ranges of the positive gravitational constant for $\kappa_{f}^{2} / \kappa_{g}^{2}=0.1$ and 10 in Fig 15

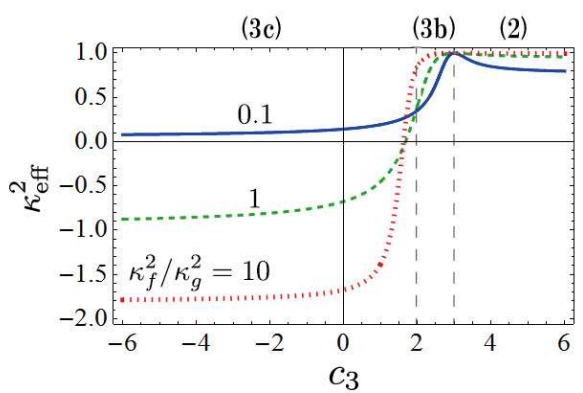

(a) Case (I)

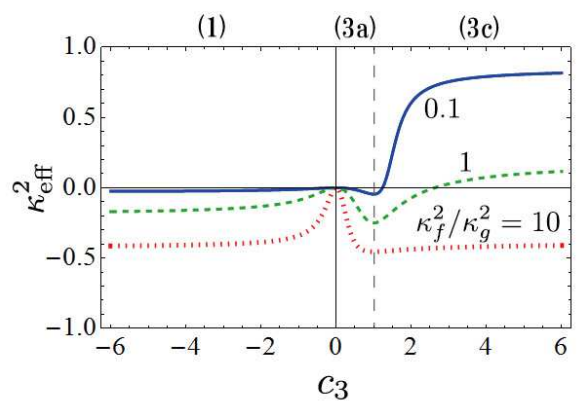

(b) Case (II)

FIG. 15: The effective gravitational constant $\kappa_{\text {eff }}^{2}$ for two oneparameter families of coupling constants ((a) $b_{0}=0$ and (b) $\left.b_{4}=0\right)$. We plot three cases of $\kappa_{f}^{2} / \kappa_{g}^{2}=0.1,1.0$, and 10 ..

for the cases (I) and (II), and the contour maps of $\kappa_{\text {eff }}^{2}$ in Fig. 16 in the $c_{3}-c_{4}$ plane. When $\kappa_{f}^{2} / \kappa_{g}^{2}$ decreases, the physically allowed region with a positive effective gravitational constant then increases in the range of $c_{3}>0$, but decreases in the range of $c_{3}<0$, and vice versa.

We may have also another constraint on the effective gravitational constant. The effective gravitational constant $\kappa_{\text {eff }}^{2}$ is different from the bare value $\kappa_{g}^{2}$. In particular, Model E with $\kappa_{f}^{2}=\kappa_{g}^{2}$ gives a big discrepancy. One may wonder whether such a discrepancy is acceptable or not. Because we know that the difference between the local gravitational constant (Newtonian gravitational constant) and the cosmological one should not be so large 57]. If the local gravitational constant is $\kappa_{g}^{2}$ or very close to it, we will find a stronger constraint on the coupling parameters $\left(c_{3}\right.$ and $\left.c_{4}\right)$ as well as the ratio of $\kappa_{f}^{2} / \kappa_{g}^{2}$. For example, for Model E, if $\kappa_{f}^{2} / \kappa_{g}^{2}<0.0366125$, we find $\kappa_{\text {eff }}^{2} / \kappa_{g}^{2}>0.9$, which may be consistent with observations. Although we expect that the local gravitational constant is close to the bare gravitational constant $\kappa_{g}^{2}$, to confirm the above constraint, we have to calculate the local gravitational constant assuming that the Vainshtein mechanism is working. 


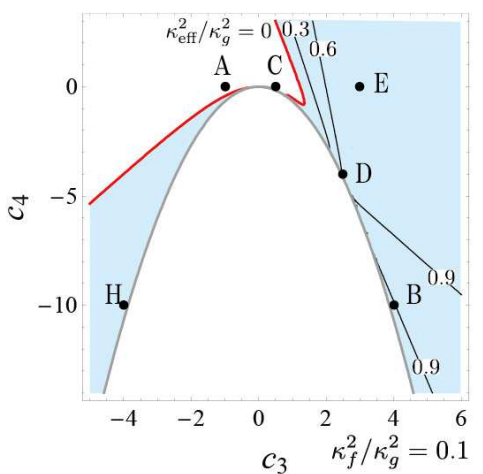

(a) $\kappa_{f}^{2} / \kappa_{g}^{2}=0.1$

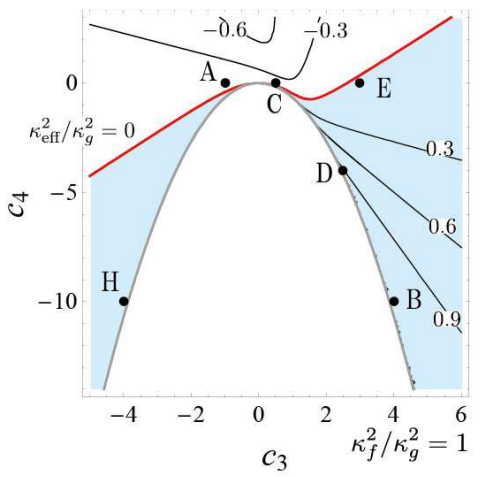

(b) $\kappa_{f}^{2} / \kappa_{g}^{2}=1$

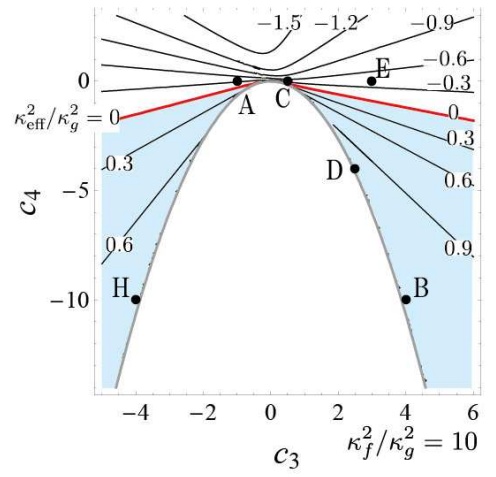

(c) $\kappa_{f}^{2} / \kappa_{g}^{2}=10$

FIG. 16: The contour maps of the effective gravitational constant $\kappa_{\text {eff }}^{2}$ in the $c_{3}-c_{4}$ plane for three cases of $\kappa_{f}^{2} / \kappa_{g}^{2}=0.1$ (a), 1.0 (b), and 10 (c). The red thick curves denote the contour of $\kappa_{\text {eff }}^{2}=0$, below which (the stripe-shaded light-blue region) we find the region of $\kappa_{\text {eff }}^{2}>0$, which is physically required.

\section{C. "Dark Matter"}

Next we discuss the possibility to explain "dark matter" component in the Friedmann equation by another one of twin matter fluids. In Table VI, we show the ratio of "dark matter" density $\rho_{D}$ to that of $g$-matter $\rho_{g, \mathrm{~m}}$ for Models B, D and E. Its value, of course, depends on the ratio $r_{\mathrm{m}}$. If $\rho_{g, \mathrm{~m}}$ consists only of a baryonic matter, it gives the ratio of dark matter to a baryonic matter, which is about 5 from the cosmic pie 1]. So choosing $r_{\mathrm{m}}$ appropriately as in Table VI we find the observed value.

\begin{tabular}{|c||c|}
\hline Model & $\rho_{\mathrm{D}} / \rho_{g}$ \\
\hline $\mathrm{B}$ & $0.16261 r_{\mathrm{m}}$ \\
\hline $\mathrm{D}$ & $0.32278 r_{\mathrm{m}}$ \\
\hline $\mathrm{E}$ & $44.9613 r_{\mathrm{m}}$ \\
\hline
\end{tabular}

TABLE VI: The ratio of the "dark sector" energy density to the matter energy density in $g$-spacetime. $r_{\mathrm{m}}$ is the ratio of twin matter energy densities.

However, in order for the de Sitter attractor to be natural, we have the constraint on $r_{\mathrm{m}}$ as we discussed in $\S$. IVB. For example, for Model B, if $r_{\mathrm{m}}<r_{\mathrm{m}}^{(\mathrm{cr})}=0.41105$, the universe approaches to de Sitter spacetime for any possible initial value $\tilde{B}\left(<\tilde{B}_{\left(\mathrm{AdS}_{2}\right)}=0.59766\right)$. This critical value gives $\rho_{D} / \rho_{g}<0.0656841$, which is too small to explain the present amount of dark matter. To find $\rho_{D} / \rho_{g} \sim 5$, we need $r_{\mathrm{m}} \sim 30$, for which a fine-tuning of initial data is required to find de Sitter universe. Similarly Model D requires a fine-tuning for de Sitter spacetime. Only Model E gives a model which explains the amount of dark matter as well as de Sitter accelerating universe, because any initial value of $\tilde{B}$ leads an de Sitter attractor, assuming $\epsilon=-1$. In this case, however, the effective gravitational constant may be too small. For example, $\kappa_{\text {eff }}^{2} / \kappa_{g}^{2}=0.0283764$ for $\kappa_{g}^{2}=\kappa_{f}^{2}$. Although this value can become close to $\kappa_{g}^{2}$ if we choose $\kappa_{f}^{2} / \kappa_{g}^{2} \ll 1$, we need a fine-tuning of initial data to find de Sitter universe. (see Fig 14 (b).)

More natural model is found if we choose the coupling constants in the left-bottom region in the $c_{3}-c_{4}$ plane. One example is Model $\mathrm{H}$ with $c_{3}=-4, c_{4}=-10$, which is plotted by the dot $\mathrm{H}$ in Fig. 16, and which data and properties are given in Table VII and in Fig. 17.

\begin{tabular}{|c|c||r|c|c|r|c|}
\hline Model & $\left(c_{3}, c_{4}\right)$ & region & $\epsilon$ & $B_{\ell}$ & $\Lambda_{g}$ & vacuum \\
\hline \hline $\mathrm{H}$ & \multirow{2}{*}{$(-4,-10)$} & \multirow{2}{*}{$(1)$} & -1 & -42.9813 & $-1.22 \times 10^{6} m_{g}^{2}$ & $\mathrm{AdS}_{1}$ \\
\cline { 4 - 7 } & & & 1 & 1 & & $\mathrm{M}$ \\
\cline { 4 - 7 } & & & 1 & 1.35254 & $-0.193237 m_{g}^{2}$ & $\mathrm{AdS}_{2}$ \\
\cline { 4 - 7 } & & & 1 & 1.84303 & $0.256594 m_{g}^{2}$ & $\mathrm{dS}$ \\
\hline
\end{tabular}

TABLE VII: $B_{\ell}$ and $\Lambda_{g}$ for Model $\mathrm{H}$.

We present two following examples for the appropriate values of $\kappa_{f}^{2} / \kappa_{g}^{2}$ and the ratio $r_{\mathrm{m}}$ :

$$
\begin{aligned}
& \text { Model HI }: \kappa_{f}^{2} / \kappa_{g}^{2}=60, r_{\mathrm{m}}=180, \\
& \text { Model HII }: \kappa_{f}^{2} / \kappa_{g}^{2}=1000, r_{\mathrm{m}}=3000 .
\end{aligned}
$$

We find $\kappa_{\text {eff }}^{2} / \kappa_{g}^{2}=0.946314$ and $\rho_{\mathrm{D}} / \rho_{g}=5.54065$ for Model HI, while $\kappa_{\text {eff }}^{2} / \kappa_{g}^{2}=0.996608$ and $\rho_{\mathrm{D}} / \rho_{g}=5.41619$ 


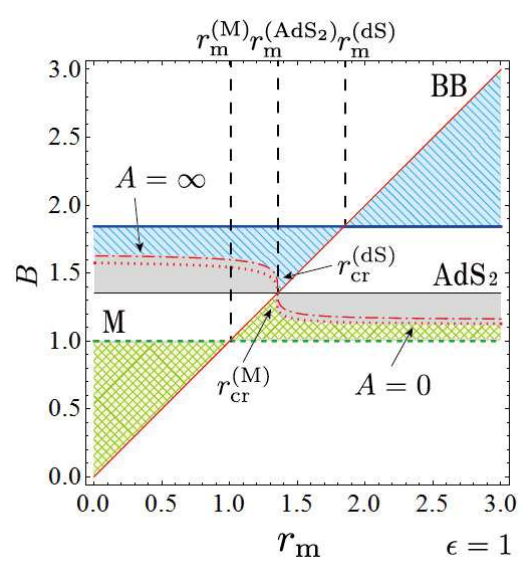

FIG. 17: The same figure as Fig. 4 for Model HI $\left(c_{3}=\right.$ $-4, c_{4}=-10$ and $\left.\kappa_{f}^{2} / \kappa_{g}^{2}=60\right)$. We have $r_{\mathrm{m}}^{\left(\mathrm{AdS}_{2}\right)}=1.35254$, $r_{\mathrm{m}}^{(\mathrm{dS})}=1.84303, r_{\mathrm{cr}}^{(\mathrm{M})}=1.34987$, and $r_{\mathrm{cr}}^{(\mathrm{dS})}=1.35313$.

for Model HII, both of which are consistent with observations.

\section{D. $\Lambda$-CDM Model}

Although the ratio of dark matter to baryonic matter is constant, their total amount is time-dependent. Hence in order to explain the present ratio of each component in the cosmic pie (the pie chart of the content of the Universe), we have to analyze the evolution of the universe. In the earlier stage of the universe, that is, when $\tilde{B}$ is not close to $\tilde{B}_{\mathrm{dS}}$, the Friedmann equation is not described by the standard form (5.6). The interaction term can not be written only by the linear combination of $\Lambda_{g}, \rho_{g}$, and $\rho_{f}$ with $\kappa_{\text {eff }}^{2}$. So we redefine the density of dark sector $\bar{\rho}_{\mathrm{D}}$ by

$$
\kappa_{\mathrm{eff}}^{2} \bar{\rho}_{\mathrm{D}}=\kappa_{g}^{2} \rho_{g}^{[\gamma]}-\Lambda_{g}-\kappa_{\mathrm{eff}}^{2} \rho_{g},
$$

which includes higher-order terms of $\left(\tilde{B}-\tilde{B}_{\mathrm{dS}}\right)$. Note that $\bar{\rho}_{\mathrm{D}} \rightarrow \rho_{\mathrm{D}}$ as $\tilde{B} \rightarrow \tilde{B}_{\mathrm{dS}}$, which provides the present amount of dark matter.

Introducing the density parameters, which are defined by

$$
\begin{aligned}
& \Omega_{\Lambda}=\frac{\Lambda_{g}}{3 H_{g}^{2}}, \quad \Omega_{\mathrm{D}}=\frac{\kappa_{\text {eff }}^{2} \bar{\rho}_{\mathrm{D}}}{3 H_{g}^{2}}, \\
& \Omega_{\mathrm{m}}=\frac{\kappa_{\text {eff }}^{2} \rho_{g, \mathrm{~m}}}{3 H_{g}^{2}}, \quad \Omega_{\mathrm{r}}=\frac{\kappa_{\text {eff }}^{2} \rho_{g, \mathrm{r}}}{3 H_{g}^{2}}, \quad \Omega_{\mathrm{k}}=-\frac{k}{H_{g}^{2}},
\end{aligned}
$$

we obtain the Friedmann equation for $g$-spacetime as

$$
\Omega_{\Lambda}+\Omega_{\mathrm{D}}+\Omega_{\mathrm{m}}+\Omega_{\mathrm{r}}+\Omega_{\mathrm{k}}=1 .
$$

From the observation, our universe is almost flat and the radiation energy is ignorable. Hence we assume that
$\Omega_{\mathrm{r}}=0$ and $\Omega_{\mathrm{k}}=0$. The present ratio of dark energy (a cosmological constant) is about $70 \%$, while that of the matter density including dark matter is about $30 \%$ [1] , i.e,

$$
\left.\Omega_{\Lambda}\right|_{0} \simeq 0.7,\left.\quad\left(\Omega_{\mathrm{D}}+\Omega_{\mathrm{m}}\right)\right|_{0} \simeq 0.3
$$

We also know that the baryonic density is given by $\left.\Omega_{\mathrm{b}}\right|_{0} \sim 0.05[1]$.

In order to analyze whether our cosmological model is consistent with the history of the universe as well as the present observations, we show the time evolution of the density parameters. We choose one successful model with the appropriate values of $\kappa_{f}^{2} / \kappa_{g}^{2}$ and $r_{\mathrm{m}}$ (Models HI and HII). In Fig. 18, we show the results for those two models. The present time, which is shown by the dashed lines in the figures, is fixed by the observed value of the deceleration parameter $q=-\ddot{a} a / \dot{a}^{2}=-0.527 \pm$ $0.026[1]$. We find that the present total matter density $\left.\left(\Omega_{\mathrm{D}}+\Omega_{\mathrm{m}}\right)\right|_{0}$ is about 0.3 and the dark energy $\left.\Omega_{\Lambda}\right|_{0}$ is about 0.7, respectively, as shown in Fig. 18. This result does not depend on the choice of initial value of $\tilde{B}$.

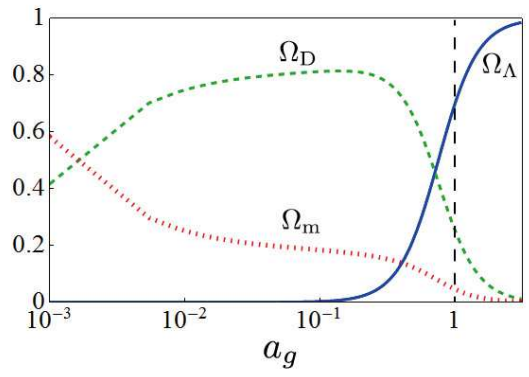

(a)

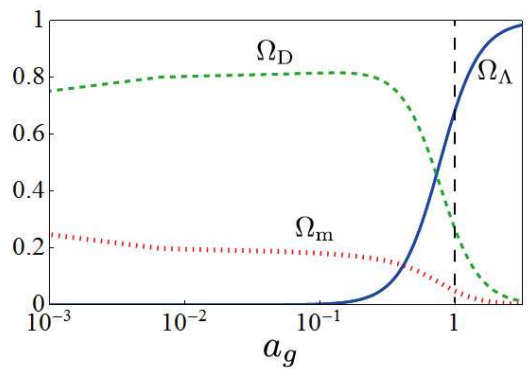

(b)

FIG. 18: The time evolution of density parameters for Model HI $\left(\kappa_{f}^{2} / \kappa_{g}^{2}=60\right.$ and $\left.r_{\mathrm{m}}=180\right)$ and for Model HII $\left(\kappa_{f}^{2} / \kappa_{g}^{2}=\right.$ 1000 and $\left.r_{\mathrm{m}}=3000\right) . a_{g}=1$ is the present time.

Since the ratio $\rho_{D} / \rho_{g} \sim 5$ for both models, we find $\left.\Omega_{\mathrm{D}}\right|_{0} \sim 0.25$ and $\left.\Omega_{\mathrm{m}}\right|_{0} \sim 0.05$, which must consist of baryonic matter because $\left.\Omega_{\mathrm{b}}\right|_{0} \sim 0.05$. We need not to introduce non-baryonic dark matter in $g$-spacetime. Another one of twin matter fluids plays a role of dark matter in the effective Friedmann equation. We should, of course, ask whether another one of twin matter fluids can 
really play a role of dark matter in the other situations such as the cosmic structure formation or the missing mass in a galactic scale. For such a purpose, we have to analyze an inhomogeneous spacetime (either perturbations or non-linear but Newtonian system).

Since the value of $\tilde{B}$ is finite in any time because of the potential form, then the interaction energy density $\rho_{g}^{[\gamma]}(\tilde{B})$ and then the dark matter density $\rho_{\mathrm{D}}$ are also finite. On the other hand, the ordinary matter density $\rho_{g}$ is proportional to $a_{g}^{-3}$ and then it dominates the universe in the early phase.

The equal time when two energy densities become the same is after recombination for Model HI while before for Model HII. For Model HI, the dark energy density is smaller than the baryonic density at recombination. This fact may show a difficulty of this model in considering the structure formation because the baryon density fluctuation at recombination era is strongly constrained by CMB observation.

We note that the above scenario will be changed if we have large amount of $f$-radiation at present. Since the dark sector $\rho_{D}$ is dominated by radiation, it does not provide "dark matter" component. Hence $\rho_{g}$ term must contain dark matter as usual scenario. $\rho_{D}$ gives just a dark radiation, which may be strongly constrained[1].

\section{CONCLUDING REMARKS}

We have studied the dynamics of homogeneous and isotropic FLRW spacetime in the ghost-free bigravity theory including twin matter sources. Assuming the coupling parameters guaranteeing the existence of de Sitter space as well as Minkowski spacetime, we find two stable attractors for spacetime with "twin" dust matter fields: One is de Sitter accelerating universe and the other is matter dominated universe. We also find the universe with a future singularity for some initial data. However a considerable number of initial data leads to de Sitter universe. Hence, although the cosmic no-hair conjecture does not exactly hold, the accelerating de Sitter universe is found naturally. The $\Lambda$-CDM model is obtained as an attractor. We also show that the dark matter component in the Friedmann equation, which originates from another twin matter, can be about 5 times larger than the baryonic matter, by choosing the appropriate coupling constants. For such a model, our matter field consists just of baryons.

One interesting remaining question is whether another twin matter can behave really as dark matter. Dark matter is required not only in the big bang scenario but also in the cosmological structure formation and as dark matter halos existing around galaxies. In order to clarify such a question, we have to analyze inhomogeneous models, either in a perturbative approach or by non-linear analysis. The linear perturbation analysis is now in progress. An- other important question is whether the bigravity theory will dynamically recover GR with/without a cosmological constant. That is, is a homothetic solution an attractor in more general spacetime? This question may be related with the above non-linear analysis. One simple analysis could be performed in a spherically symmetric system. A spherical static spacetime including a black hole has also been studied both in the massive gravity and bigravity theories 58 62]. Although the perturbation analysis show the existence of some instability [63, 64], since the time scale is about the age of the present universe, we are interested in whether we find a homothetic solution (GR) in a local dynamical free-fall time scale. It is under investigation.

\section{Acknowledgments}

We would like to thank Takahiro Tanaka for useful discussions. This work was supported in part by Grantsin-Aid from the Scientific Research Fund of the Japan Society for the Promotion of Science (No. 25400276). 
[1] P. Ade et al. (Planck Collaboration), arXive:1303.5076.

[2] S. Perlmuter et al. Astrophys. J. 517, 565 (1999) astro-ph/9812133.

[3] A. G. Riess et al. Astron. J. 116, 1009 (1998) astro-ph/9805201.

[4] M. Fierz and W. Paui, Proc. Roy. Soc. Lond. A 173, 211 (1939).

[5] D. G. Boulware and S. Deser, Phys. Rev. D 6, 3368 (1972).

[6] C. de Rham, G. Gabadadze and A. J. Tolley, Phys. Rev. Lett. 106, 231101 (2011).

[7] S. F. Hassan and R. A. Rosen, Phys. Rev. Lett. 108, 041101 (2012).

[8] A. Golovnev, Phy. Lett. B 707 (2012) 404-408.

[9] S. F. Hassan and R. A. Rosen, J. High Energy Phys. 04 (2012) 123.

[10] S. F. Hassan, A. Schmidt-May, M. von Strauss, Phys. Lett. B 715 (2012) 335-339.

[11] J. Klusoň, Phys. Rev. D 86, 044024 (2012).

[12] Recently, however, another possible issue on superluminality and acausality has been argued in

S. Deser, M. Sandora and A. Waldron, Phys. Rev D 88, 081501 (2013); Phys. Rev D 87, 101501 (2013). K. Izumi and Y. C. Ong, Classical Quant. Gravity, 30, 184008 (2013). S. Deser, K. Izumi, Y. C. Ong and A. Waldron, Phys. Lett. B 726 (2013) 544-548; arXiv:1312.1115

[13] S. F. Hassan and R. A. Rosen, J. High Energy Phys. 02 (2012) 126.

[14] G. DfAmico, C. de Rham, S. Dubovsky, G. Gabadadze, D. Pirtskhalava and A. J. Tolley, Phys. Rev. D 84, 124046 (2011)

[15] A. E. Gümrükçüoğlu, C. Lin, S. Mukohyama, J. Cosmol. Astropart. Phys. 1111 (2011) 030.

[16] P. Gratia, W. Hu, and M. Wyman, Phys.Rev. D86, 061504 (2012).

[17] T. Kobayashi, M. Siino, M. Yamaguchi, and D. Yoshida, Phys.Rev. D86, 061505 (2012).

[18] A. H. Chamseddine, M. S. Volkov, Phys. Lett. B 704 (2011) 652-654.

[19] M. S. Volkov, Phys. Rev. D 86, 061502 (2012).

[20] M. S. Volkov, Phys. Rev. D 86, 104022 (2012).

[21] A. De Felice, A. E. Gümrükçüoğlu, S. Mukohyama, Phys.Rev.Lett. 109 (2012) 171101.

[22] A. E. Gümrükçüoğlu, C. Lin, S. Mukohyama, Phys. Lett. B 717 (2012) 295-298.

[23] T. Q. Do and W. F. Kao, Phys. Rev. D 88, 063006 (2013).

[24] M. S. Volkov, J. High Energy Phys. 1201 (2012) 035.

[25] M. von Strauss, A. Schmidt-May, J. Enander, E. Mortsell, and S. F. Hassan, J. Cosmol. Astropart. Phys. 1203 (2012) 042.

[26] D. Comelli, M. Crisostomi, F. Nesti and L. Pilo, J. High Energy Phys. 03 (2012) 067.

[27] Y. Akrami, T. S. Koivisto, M. Sandstad, J. High Energy Phys. 1303 (2013) 099.

[28] Y. Akrami, T. S. Koivisto, D. F. Mota, and M. Sandstad, J. Cosmol. Astropart. Phys. 1310 (2013) 046.

[29] Y. Sakakihara, J. Soda, and T. Takahashi, PTEP 2013 033E02 (2013).

[30] S. Capozziello and P. Martin-Moruno, Phys. Lett. B 719 (2013) 14-17.

[31] D. Comelli, M.Crisostomi and L. Pilo, J. High Energy
Phys. 06 (2012) 085.

[32] N. Khosravi, H. R. Sepangi and S. Shahidi, Phys. Rev. D 86, 043517 (2012)

[33] M. Berg, I. Buchberger, J. Enander, E. Mörtsell and S. Sjörs, J. Cosmol. Astropart. Phys. 1212 (2012) 021.

[34] M. Fasiello and J. Tolley, J. Cosmol. Astropart. Phys. 1211 (2012) 035; M. Fasiello and J. Tolley, J. Cosmol. Astropart. Phys. 12 (2013) 002.

[35] K. Maeda and M. S. Volkov, Phys. Rev. D 87, 104009 (2013).

[36] See, for example, C. Will, Living Rev. Rel. 9,3 (2005)

[37] M. Milgrom, Mon. Not. R. Astron. Soc. 405, 1129 (2010).

[38] G. W. Gibbons and S. W. Hawking, Phys. Rev. D 15, 2738 (1977); S. W. Hawking and I. G. Moss, Phys. Lett. B108, 35 (1982)

[39] R. M. Wald, Phys. Rev. D 28, 2118 (1983).

[40] A. A. Starobinsky, JETP Lett. 30, 682 (1979); A. A. Starobinsky, JETP Lett. 37, 55 (1983).

[41] J. D. Barrow and J. Stein-Schabes, Phys. Lett. A 103, 315 (1984)

[42] Y. Kitada, K. Maeda Class.Quant.Grav. 10,703 (1993); Y. Kitada, K. Maeda Phys.Rev. D45,1416 (1992).

[43] K. Nakao, T. Shiromizu, K. Maeda Class.Quant.Grav. 11, 2059 (1994); T. Shiromizu, K. Nakao, H. Kodama, K. Maeda, Phys.Rev. D47, 3099 (1993).

[44] K. Nakao, T. Nakamura, K. Oohara, K. Maeda, Phys.Rev. D43, 1788 (1991);7 K. Nakao, T. Nakamura, K. Maeda, K. Oohara Phys.Rev. D47, 3194 (1993); M. Shibata, K. Nakao, T. Nakamura, K. Maeda, Phys.Rev. D50, 708 (1994).

[45] C. J. Isham , A. Salam , J. A. Strathdee, Phys. Rev. D, 3, 867 (1971).

[46] N. Rosen , Ann. Phys., 84, 455 (1974).

[47] N. Boulanger, T. Damour, L. Gualtieri, M. Henneaux, Nuclear Phys. B 597, 127 (2001).

[48] T. Damour, I. I. Kogan, Phys. Rev. D 66, 104024 (2002).

[49] D. Blas, C. Deffayet, J. Garriga, Classical Quant. Gravity, 23, 1697 (2006).

[50] M. Bañados, Ferreira, C. Skordis, Phys. Rev. D, 79, 063511 (2009); M. Banados, A. Gomberoff, D. C. Rodrigues, C. Skordis, Phys. Rev. D 79, 063515 (2009).

[51] We call the spacetime with $g$-metric a $g$-spacetime, while one with $f$-metric a $f$-spacetime.

[52] A. Higuchi, Nucl. Phys. B 282 (1987) 397.

[53] A. Higuchi, Nucl. Phys. B 325 (1989) 745.

[54] S. Deser and A. Waldron, Phys. Rev. Lett. 87, 031601(2001); S. Deser and A. Waldron, Nucl. Phys. B 607 (2001) 577.

[55] S. F. Hassan, A. Schmidt-May, and M. von Strauss, Phys Lett. B726 (2013) 834-838.

[56] Assuming that the $g$-metric is the FLRW type but the $f$-metric is just spherically symmetric, we find two cases: one is both metrics are diagonal as we assumed here, and the other is $f$-metric is non-diagonal. The latter case turns out to be both $g$-and $f$-metrics satisfy the conventional Einstein equations with a cosmological constant. Since we know FLRW universe in GR very well, we do not discuss this case here.

[57] See, for example, J.-P. Uzan, Rev. Mod. Phys. 75, 403 (2003).

[58] Th. M. Nieuwenhuizen, Phys.Rev.D84, 024038 (2011). 
[59] K. Koyama, G. Niz and G. Tasinato, Phys. Rev. Lett. 107, 131101 (2011); K. Koyama, G. Niz and G. Tasinato, Phys. Rev. D 84, 064033 (2011).

[60] M. S. Volkov, Phys. Rev. D 85, 124043 (2012).

[61] V. Baccetti, P.Martin-Moruno and M. Visser, J. High Energy Phys. 08, 108 (2012).

[62] E. Babichev and M. Crisostomi, Phys. Rev. D 88, 084002
(2013).

[63] E. Babichev and A. Fabbri, Classical Quantum Gravity 30, 152001 (2013).

[64] R. Brito, V. Cardoso and P. Pani, Phys. Rev. D 88, 023514 (2013).

\section{Appendix A: The perturbations around the homothetic solution}

Since the homothetic spacetimes are given by the solutions in GR, such solutions are important if they are stable. So we shall discuss the perturbations around such a homothetic solution. The basic equations are Eqs. (2.20) and (2.21).

The unperturbed spacetimes are assumed to be homothetic, i.e.,

$$
\stackrel{(0)}{g}_{\mu \nu}, \text { and } \stackrel{(0)}{f}_{\mu \nu}=K^{2} \stackrel{(0)}{g}_{\mu \nu}
$$

which are the solutions of

$$
\begin{aligned}
& \stackrel{(0)}{G^{\mu}}{ }_{\nu}(\stackrel{(0)}{g})=-\Lambda_{g}(K) \delta^{\mu}{ }_{\nu}+\kappa_{g}^{2} \stackrel{(0)}{T}^{[\mathrm{m}] \mu}{ }_{\nu}, \\
& \stackrel{(0)}{\mathcal{G}}^{\mu}{ }_{\nu}(\stackrel{(0)}{f})=-\Lambda_{f}(K) \delta^{\mu}{ }_{\nu}+\kappa_{f}^{2} \stackrel{(0)}{\mathcal{T}}^{[\mathrm{m}] \mu}{ }_{\nu},
\end{aligned}
$$

A constant $K$ is determined by a solution of Eq. (2.31), and

$$
\kappa_{f}^{2} \stackrel{(0)}{\mathcal{T}}_{[m] \mu}{ }_{\nu}=\frac{1}{K^{2}} \kappa_{g}^{2} \stackrel{(0)}{T}_{\nu}^{[m] \mu}{ }_{\nu}
$$

We then consider the following perturbations:

$$
\begin{aligned}
& g_{\mu \nu}=\stackrel{(0)}{g}_{\mu \nu}+\epsilon h_{\mu \nu} \\
& f_{\mu \nu}=K^{2} \tilde{f}_{\mu \nu}=K^{2}\left(\stackrel{(0)}{g}_{\mu \nu}+\epsilon k_{\mu \nu}\right)
\end{aligned}
$$

where $\epsilon \ll 1$. The sufficies of $k_{\mu \nu}$ as well as $h_{\mu \nu}$ are moved by the background metric $\stackrel{(0)}{g}_{\mu \nu}$.

The energy-momentum tensors of twin matter fluid and those from the interaction terms can be expanded as

$$
\begin{aligned}
\kappa_{g}^{2} T_{\nu}^{[m] \mu} & =\kappa_{g}^{2}\left[{\left.\stackrel{(0)}{T} \underset{\nu}{[m] \mu}+\epsilon \stackrel{(1)}{T}_{\nu}^{[m] \mu}+\cdots\right]}_{\kappa_{f} \mathcal{T}_{\nu}^{[m] \mu}}=\kappa_{f}^{2}\left[\stackrel{(0)}{\mathcal{T}}[m] \mu_{\nu}+\epsilon \stackrel{(1)}{\mathcal{T}}[m] \mu_{\nu}+\cdots\right]\right.
\end{aligned}
$$

and

respectively, where

$$
\begin{aligned}
\kappa_{g}^{2} T_{\nu}^{[\gamma] \mu} & =-\Lambda_{g} \delta^{\mu}{ }_{\nu}+\epsilon \kappa_{g}^{2}{ }^{(1)}{ }^{[\gamma] \mu}{ }_{\nu} \\
\kappa_{f}^{2} \mathcal{T}^{[\gamma] \mu}{ }_{\nu} & =-\Lambda_{f} \delta^{\mu}{ }_{\nu}+\epsilon \kappa_{f}^{2}{ }_{\mathcal{T}}^{(1)}{ }_{\nu}^{[\gamma] \mu},
\end{aligned}
$$

$$
\begin{aligned}
\kappa_{g}^{2} \stackrel{(1)}{T}_{\nu}^{[\gamma] \mu} & =m_{g}^{2}\left[\stackrel{(1)}{\tau}_{\nu}{ }_{\nu}(h, k)-\delta \stackrel{(0)}{\mathscr{U}}(h, k) \delta_{\nu}^{\mu}\right] \\
\kappa_{f}^{2} \stackrel{(1)}{\mathcal{T}}[\gamma] \mu_{\nu} & =-\frac{m_{f}^{2}}{K^{4}}\left[(h-k) \stackrel{(1)}{\tau} \mu_{\nu}+\stackrel{(1)}{\tau}_{\mu}{ }_{\nu}(h, k)\right]
\end{aligned}
$$

with

$$
\begin{aligned}
\stackrel{(1)}{\tau}_{\nu}{ }_{\nu}= & -\frac{1}{2}\left[\left(b_{1} K+2 b_{2} K^{2}+b_{3} K^{3}\right)\left(h^{\mu}{ }_{\nu}-k_{\nu}^{\mu}\right)\right. \\
& \left.+\left(b_{2} K^{2}+2 b_{3} K^{3}+b_{4} K^{4}\right)(h-k) \delta^{\mu}{ }_{\nu}\right] \\
\dot{(0)}^{(0)}= & -\frac{1}{2}\left(b_{1} K+3 b_{2} K^{2}+3 b_{3} K^{3}+b_{4} K^{4}\right)(h-k), \\
h= & h^{\alpha}{ }_{\alpha}, \quad k=k_{\alpha}^{\alpha} .
\end{aligned}
$$

The first order perturbation equations are given by:

$$
\begin{aligned}
& \stackrel{(0)}{g} \mu \rho \stackrel{(1)}{R}_{\rho \nu}(\varphi)-\stackrel{(0)}{R}^{\rho(\mu} \varphi_{\nu) \rho}=\stackrel{(1)}{M}^{[\mathrm{m}] \mu}{ }_{\nu}-\frac{1}{4} m_{\mathrm{eff}}^{2}\left[2 \varphi^{\mu}{ }_{\nu}+\varphi \delta_{\nu}^{\mu}\right], \\
& \stackrel{(0)}{g}^{\mu \rho} \stackrel{(1)}{R}_{\rho \nu}(\psi)-\stackrel{(0)}{R}^{\rho(\mu} \psi_{\nu) \rho}=\stackrel{(1)}{\mathcal{M}}^{[\mathrm{m}] \mu}{ }_{\nu},
\end{aligned}
$$

where we have introduced new variables $\varphi_{\mu \nu}$ and $\psi_{\mu \nu}$ from two metric perturbations as

$$
\varphi_{\mu \nu}:=h_{\mu \nu}-k_{\mu \nu}, \quad \psi_{\mu \nu}:=m_{f}^{2} h_{\mu \nu}+K^{2} m_{g}^{2} k_{\mu \nu}
$$


and defined by

$$
\begin{aligned}
& \stackrel{(1)}{M}_{\nu}^{[\mathrm{m}] \mu}{ }_{\nu}=\kappa_{g}^{2}\left[\stackrel{(1)}{T}^{[\mathrm{m}] \mu}{ }_{\nu}-\frac{1}{2} \stackrel{(1)}{T}^{[\mathrm{m}]} \delta^{\mu}{ }_{\nu}\right]-K^{2} \kappa_{f}^{2}\left[\stackrel{(1)}{\mathcal{T}}[\mathrm{m}] \mu_{\nu}-\frac{1}{2} \stackrel{(1)}{\mathcal{T}}^{[\mathrm{m}]} \delta^{\mu}{ }_{\nu}\right] \\
& \stackrel{(1)}{\mathcal{M}}^{[\mathrm{m}] \mu}{ }_{\nu}=\frac{\kappa_{g}^{2} \kappa_{f}^{2}}{\kappa^{2}} m^{2}\left[\left(\stackrel{(1)}{T}^{[\mathrm{m}] \mu}{ }_{\nu}-\frac{1}{2} \stackrel{(1)}{T}^{[\mathrm{m}]} \delta^{\mu}{ }_{\nu}\right)+K^{4}\left(\stackrel{(1)}{\mathcal{T}}[\mathrm{m}] \mu_{\nu}-\frac{1}{2}{ }^{(1)}{ }^{[m]} \delta^{\mu}{ }_{\nu}\right)\right] .
\end{aligned}
$$

$\psi_{\mu \nu}$ and $\varphi_{\mu \nu}$ describe a massless and massive modes, respectively. $m_{\text {eff }}$ denotes graviton mass of the massive mode in the homothetic background spacetime, which is given by

$$
m_{\mathrm{eff}}^{2}=\left(m_{g}^{2}+\frac{m_{f}^{2}}{K^{2}}\right)\left(b_{1} K+2 b_{2} K^{2}+b_{3} K^{3}\right) .
$$

If the background is the Minkwoski spacetime $(K=1)$, we find $m_{\mathrm{eff}}=m$.

$\stackrel{(1)}{R}_{\mu \nu}$ is the linear perturbation operator of the Ricci tensor, which is defined for metric perturbation $h_{\mu \nu}$ by

$$
\stackrel{(1)}{R}_{\mu \nu}(h)=\frac{1}{2}\left[-\stackrel{(0)}{\nabla} \stackrel{(0)}{\nabla}_{\nu} h-\stackrel{(0)}{\square} h_{\mu \nu}+\stackrel{(0)}{\nabla}^{\alpha}\left(\stackrel{(0)}{\nabla}_{\nu} h_{\alpha \mu}\right)+\stackrel{(0)}{\nabla}^{\alpha}\left({ }^{(0)} \nabla_{\mu} h_{\alpha \nu}\right)\right] .
$$

The Bianchi identity $\left(\nabla_{\mu} G^{\mu}{ }_{\nu}=0\right)$ gives the conservation of "graviton" $\gamma^{\mu}{ }_{\nu}$, i.e.,

$$
\nabla_{\mu} T_{\nu}^{[\gamma] \mu}=0
$$

which perturbation gives the constraint on the massive mode $\varphi_{\alpha \beta}$ :

$$
\stackrel{(0)}{\nabla}{ }_{\mu} \kappa_{g}^{2} \stackrel{(1)}{T}_{[\gamma] \mu}{ }_{\nu}=\frac{m_{g}^{2}}{2}\left(b_{1} K+2 b_{2} K^{2}+b_{3} K^{3}\right)\left[-\stackrel{(0)}{\nabla}{ }_{\mu} \varphi^{\mu}{ }_{\nu}+\stackrel{(0)}{\nabla}{ }_{\nu} \varphi\right]=0
$$

Since $m_{\text {eff }}^{2} \neq 0$, we find

$$
\stackrel{(0)}{\nabla}_{\mu} \varphi_{\nu}^{\mu}=\stackrel{(0)}{\nabla}_{\nu} \varphi
$$

Taking a trace of Eq. (A12) and using Eq. (A20), we find

$$
\left(3 m_{\mathrm{eff}}^{2}-2 \Lambda_{g}\right) \varphi=\kappa_{g}^{2}\left(2 \stackrel{(0)}{T}_{\alpha \beta}^{[\mathrm{m}]} \varphi^{\alpha \beta}-\stackrel{(0)}{T}_{[\mathrm{m}]} \varphi\right)+2 \stackrel{(1)}{[\mathrm{m}] \alpha}_{\alpha} .
$$

Eqs. A20 and A21 give five constraint equations on $\varphi_{\alpha \beta}$, which is consistent with five degrees of freedom for the massive gravition mode. There is no gauge freedom because $\varphi_{\alpha \beta}$ is a gauge invariant tensor.

Using these constraints, we rewrite the above perturbation equations as

$$
\begin{aligned}
& \stackrel{(0)}{\nabla}_{\mu} \stackrel{(0)}{\nabla}_{\nu} \varphi-\stackrel{(0)}{\square} \varphi_{\mu \nu}-2 \stackrel{(0)}{R}_{\mu}^{\alpha}{ }_{\nu}^{\beta} \varphi_{\alpha \beta}+m_{\mathrm{eff}}^{2}\left(\varphi_{\mu \nu}+\frac{1}{2} \varphi \stackrel{(0)}{g}_{\mu \nu}\right)=2 \stackrel{(1)}{M}_{\mu \nu}^{[\mathrm{m}]}, \\
& -\stackrel{(0)}{\nabla} \stackrel{(0)}{\nabla}_{\nu} \psi-\stackrel{(0)}{\square} \psi_{\mu \nu}+2 \stackrel{(0)}{\nabla}_{(\nu}\left[\stackrel{(0)}{\nabla}^{\alpha} \psi_{\mu) \alpha}\right]-2 \stackrel{(0)}{R}_{\mu}{ }^{\alpha}{ }_{\nu}{ }^{\beta} \psi_{\alpha \beta}=2 \mathcal{M}_{\mu \nu}^{(1)}[\mathrm{m}],
\end{aligned}
$$

where we have used

$$
\stackrel{(0)}{\nabla}^{\alpha}\left(\stackrel{(0)}{\nabla}_{\nu} \chi_{\mu \alpha}\right)=\stackrel{(0)}{\nabla}_{\nu}\left(\stackrel{(0)}{\nabla}^{\alpha} \chi_{\mu \alpha}\right)+\stackrel{(0)}{R}_{\mu}^{\alpha \beta}{ }_{\nu} \chi_{\alpha \beta}+\stackrel{(0)}{R}_{\nu}^{\rho} \chi_{\mu \rho}
$$

We shall discuss two decoupled modes separately.

\section{1. massless mode $\psi_{\mu \nu}$}

Introducing new variable by

$$
\bar{\psi}_{\mu \nu}=\psi_{\mu \nu}-\frac{1}{2} \psi^{(0)} \stackrel{p}{\mu \nu}_{\mu}
$$

and imposing the transverse-traceless conditions by use of gauge freedom;

$$
\begin{aligned}
& \stackrel{(0)}{\nabla}^{\mu} \bar{\psi}_{\mu \nu}=0 \\
& \bar{\psi}=0
\end{aligned}
$$


we find the perturbation equation as

$$
\stackrel{(0)}{\square} \bar{\psi}_{\mu \nu}^{(\mathrm{TT})}+2 \stackrel{(0)}{R}_{\mu}{ }^{\alpha}{ }_{\nu} \beta \bar{\psi}_{\alpha \beta}^{(\mathrm{TT})}=-2 \stackrel{(1)}{\mathcal{M}}_{\mu \nu}^{[\mathrm{m}]} .
$$

In the case of a vacuum spacetime, i.e.,

$$
\begin{aligned}
& \stackrel{(0)}{R}^{\mu}{ }_{\nu}=\Lambda_{g} \delta^{\mu}{ }_{\nu}, \quad \stackrel{(0)}{R}=4 \Lambda_{g} \\
& \stackrel{(1)}{\mathcal{M}}_{\mu \nu}^{[\mathrm{m}]}=0 .
\end{aligned}
$$

We obtain the perturbation equation as

$$
\stackrel{(0)}{\square} \bar{\psi}_{\mu \nu}^{(\mathrm{TT})}+2 \stackrel{(0)}{C}_{\mu}^{\alpha}{ }_{\nu}{ }^{\beta} \bar{\psi}_{\alpha \beta}^{(\mathrm{TT})}-\frac{2}{3} \Lambda_{g} \bar{\psi}_{\mu \nu}^{(\mathrm{TT})}=0 .
$$

If the background is de Sitter spacetime (or a spacetime without a Weyl tensor), we find

$$
\stackrel{(0)}{\square} \bar{\psi}_{\mu \nu}^{(\mathrm{TT})}-\frac{2}{3} \Lambda_{g} \bar{\psi}_{\mu \nu}^{(\mathrm{TT})}=0 .
$$

Then we can show that de Sitter expanding spacetime is stable against this perturbation mode [40].

\section{2. masslive mode $\varphi_{\mu \nu}$}

In the case of vacuum state with Eq. (A28), the trace equation (A21) is now

$$
\left(3 m_{\mathrm{eff}}^{2}-2 \Lambda_{g}\right) \varphi=0 .
$$

If $3 m_{\text {eff }}^{2} \neq 2 \Lambda_{g}$, we find $\varphi=0$. The massive mode $\varphi_{\mu \nu}$ must satisfy the transverse and traceless conditions (A20) and (A31):

$$
\stackrel{(0)}{\nabla}^{\mu} \varphi_{\mu \nu}=0, \quad \varphi=0 .
$$

The perturbation equation is now

$$
\begin{aligned}
\stackrel{(0)}{\square} \varphi_{\mu \nu} & +2 \stackrel{(0)}{C_{\mu}}{ }^{\alpha}{ }_{\nu}{ }^{\beta} \varphi_{\alpha \beta} \\
& -\left[\frac{2}{3} \Lambda_{g}+m_{\mathrm{eff}}^{2}\right] \varphi_{\mu \nu}=0
\end{aligned}
$$

We can show that de Sitter expanding spacetime (with zero Weyl curvature) is stable against the massive perturbation mode too.

The case of $3 m_{\text {eff }}^{2}=2 \Lambda_{g}$ is called "partially massless", which contains an additional gauge freedom. There are only four propagation modes [55].

\section{Appendix B: Cosmology in partially massless theory}

In this Appendix, we discuss cosmology in partially massless (PM) bigravity theory. In the PM theory, all coefficients of $C_{\Lambda}(\tilde{B})$ vanish. Then we have to deal with this case separately. However it turns out to be simpler than the general case.

The coupling constants can be described by only one free parameter $b_{2}$ such that

$$
b_{1}=b_{3}=0, \quad b_{0}=3 b_{2} \kappa_{f}^{2} / \kappa_{g}^{2}, \quad b_{4}=3 b_{2} \kappa_{g}^{2} / \kappa_{f}^{2} .
$$

By use of the relation (B1), we find the Friedmann equation as

$$
H_{g}^{2}+\frac{k}{a_{g}^{2}}=\frac{\kappa_{g}^{2}}{3}\left(\rho_{g}^{[\gamma]}+\rho_{g}\right),
$$

where $\rho_{g}$ is the ordinary matter energy density. The interaction term gives the energy density of "gravtion", $\rho_{g}^{[\gamma]}$, which is given by

$$
\rho_{g}^{[\gamma]}=\frac{3 m^{2} b_{2}}{\kappa^{2}}\left(\tilde{B}^{2}+\frac{\kappa_{f}^{2}}{\kappa_{g}^{2}}\right) .
$$

Since the coupling constant $b_{2}$ always appears with $\mathrm{m}^{2}$, it can be absorbed into the definition of $m$, fixing the constant as $b_{2}=1$ in the PM bigravity theory.

The algebraic relation (3.21) between $a_{g}$ and $\tilde{B}$ becomes

$$
\tilde{B} C_{\mathrm{m}}(\tilde{B}) a_{g}+C_{\mathrm{r}}(\tilde{B})=0,
$$

which gives

$$
\begin{aligned}
& \tilde{B}=\tilde{B}_{ \pm} \\
& := \pm \frac{c_{f, m} a_{g}+\sqrt{c_{f, m}^{2} a_{g}^{2}+4 c_{f, r}\left(c_{g, m} a_{g}+c_{g, r}\right)}}{2\left(c_{g, m} a_{g}+c_{g, r}\right)}
\end{aligned}
$$

where we have used the positivity conditions of $c_{g, \mathrm{~m}}, c_{g, \mathrm{r}}$, $c_{f, \mathrm{~m}}$, and $c_{f, \mathrm{r}}$. In order to write down the effective Friedmann equation from Eq. (B2), assuming the universe is expanding, we expand Eq. (B5) as

$$
\begin{aligned}
\tilde{B}_{ \pm}= & \pm\left[\frac{c_{f, \mathrm{~m}}}{c_{g, \mathrm{~m}}}+\left(\frac{c_{f, \mathrm{r}}}{c_{f, \mathrm{~m}}}-\frac{c_{f, \mathrm{~m}} c_{g, \mathrm{r}}}{c_{g, \mathrm{~m}}^{2}}\right) \frac{1}{a_{g}}\right. \\
& +\left(-\frac{c_{f, \mathrm{r}}^{2} c_{g, \mathrm{~m}}}{c_{f, \mathrm{~m}}^{3}}+\frac{c_{f, \mathrm{~m}} c_{g, \mathrm{r}}^{2}}{c_{g, \mathrm{~m}}^{3}}\right) \frac{1}{a_{g}^{2}} \\
& +\left(\frac{2 c_{f, \mathrm{r}}^{3} c_{g, \mathrm{~m}}^{2}}{c_{f, \mathrm{~m}}^{5}}-\frac{c_{f, \mathrm{r}}^{2} c_{g, \mathrm{r}}}{c_{f, \mathrm{~m}}^{3}}-\frac{c_{f, \mathrm{~m}} c_{g, \mathrm{r}}^{3}}{c_{g, \mathrm{~m}}^{4}}\right) \frac{1}{a_{g}^{3}} \\
& \left.+O\left(\frac{1}{a_{g}^{4}}\right)\right]
\end{aligned}
$$

as $a_{g} \rightarrow \infty$. Using this equation, we find the effective Friedmann equation as

$$
H_{g}^{2}+\frac{k}{a_{g}^{2}} \approx \frac{\Lambda_{g}}{3}+\frac{\kappa_{g}^{2}}{3}\left(\rho_{g, \mathrm{~m}}+\rho_{1}+\rho_{2}+\rho_{3}\right)
$$


where

$$
\begin{aligned}
\Lambda_{g} & =3 m_{g}^{2}\left(r_{\mathrm{m}}^{2}+\frac{\kappa_{f}^{2}}{\kappa_{g}^{2}}\right) \\
\kappa_{g}^{2} \rho_{1} & =2\left(r_{\mathrm{r}}-r_{\mathrm{m}}^{2}\right)\left(\frac{c_{g, \mathrm{r}}}{c_{g, \mathrm{~m}}}\right) \frac{1}{a_{g}} \\
\kappa_{g}^{2} \rho_{2} & =\left[\frac{\left(-r_{\mathrm{r}}+r_{\mathrm{m}}^{2}\right)\left(r_{\mathrm{r}}+3 r_{\mathrm{m}}^{2}\right)}{r_{\mathrm{m}}^{2}}\right]\left(\frac{c_{g, \mathrm{r}}}{c_{g, \mathrm{~m}}}\right)^{2} \frac{1}{a_{g}^{2}} \\
\kappa_{g}^{2} \rho_{3} & =2\left[\frac{\left(r_{\mathrm{r}}-r_{\mathrm{m}}^{2}\right)\left(r_{\mathrm{r}}^{2}+r_{\mathrm{r}} r_{\mathrm{m}}^{2}+2 r_{\mathrm{m}}^{4}\right)}{r_{\mathrm{m}}^{4}}\right]\left(\frac{c_{g, \mathrm{r}}}{c_{g, \mathrm{~m}}}\right)^{3} \frac{1}{a_{g}^{3}},
\end{aligned}
$$

with $r_{\mathrm{m}}=c_{f, \mathrm{~m}} / c_{g, \mathrm{~m}}$ and $r_{\mathrm{r}}=c_{f, \mathrm{r}} / c_{g, \mathrm{r}} . \quad \rho_{3}$ is an additional matter, $\rho_{2}$ behaves as a correction of the curvature term, and $\rho_{1}$ describes unusual matter with the equation of state $P=-\frac{2}{3} \rho$. Since the $\Lambda$-CDM model with zero spatial curvature describes the present universe very well, the last two terms ( $\rho_{1}$ and $\rho_{2}$ ) must be very small. Such a condition gives a strong constraint on both radiation components. In particular, when we can ignore the radiation terms, we find the $\Lambda$-CDM model. No additional dust component comes from the $f$-spacetime matter. Dark matter must be found in $\rho_{g, \mathrm{~m}}$. Another one of twin matters is not regarded as dark matter. The cosmological constant $\Lambda_{g}$, however in this case, depends on the ratio of twin matter fluids $r_{\mathrm{m}}$. If $\kappa_{f} \ll \kappa_{g}$, then $\Lambda \approx 3 m_{g}^{2} r_{\mathrm{m}}^{2}$. The cosmological constant depends on matter fluids. It may give us a hint to solve the socalled "considence problem", which is a mystery why the amount of dark energy is close to that of matter fluid. 